\title{
Personality differences in brain network mechanisms for placebo analgesia and nocebo hyperalgesia in experimental pain: a functional magnetic resonance imaging study
}

\author{
Yu Shi ${ }^{1 \#}$, Yaping Wang ${ }^{1 \#}$, Yanyan Zeng ${ }^{1 \#}$, Hongrui Zhan ${ }^{1,2}$, Shimin Huang ${ }^{1}$, Guiyuan Cai ${ }^{1}$, Jianming \\ Yang $^{3}$, Wen Wu ${ }^{1}$ \\ ${ }^{1}$ Department of Rehabilitation, Zhujiang Hospital, Southern Medical University, Guangzhou, China; ${ }^{2}$ Department of Physical Medicine and \\ Rehabilitation, The Fifth Affiliated Hospital of Sun Yat-sen University, Zhuhai, China; ${ }^{3}$ Department of Radiology, Zhujiang Hospital, Southern \\ Medical University, Guangzhou, China \\ Contributions: (I) Conception and design: Y Shi, W Wu; (II) Administrative support: J Yang, W Wu; (III) Provision of study materials or patients: Y \\ Shi, Y Yang, W Wu; (IV) Collection and assembly of data: Y Shi, Y Zeng, H Zhan; (V) Data analysis and interpretation: Y Shi, G Cai, S Huang; (VI) \\ Manuscript writing: All authors; (VII) Final approval of manuscript: All authors. \\ \#These authors contributed equally to this work. \\ Correspondence to: Wen Wu, MD. Department of Rehabilitation, Zhujiang Hospital, Southern Medical University, Guangzhou 510282, China. \\ Email:wuwen66@163.com.
}

Background: Placebo and nocebo responses have been increasingly gaining the attention of clinical and scientific researchers. Inconsistent conclusions from current studies indicate that different factors potentially affect both placebo and nocebo responses. Increasing evidence suggests that personality differences may affect the mechanisms of both two responses. In the present work, we explored the characteristics of neural signals of placebo and nocebo responses based on functional connectivity (FC) analysis and Granger causality analysis (GCA).

Methods: A total of 34 healthy participants received conditional induction training to establish placebo and nocebo responses. Every participant completed the following experimental workflow, including scanning of baseline, experimental low back pain model establishment, scanning of acute pain status, and scanning of placebo response or nocebo response. We collect visual analogue scale (VAS) data after each scanning. Functional magnetic resonance imaging (fMRI) data from different personality groups were subjected to FC analysis and multivariate GCA (mGCA).

Results: Pain scores for placebo and nocebo responses were statistically different across different personality. There are also statistically differences in the neural signals of two responses across different personality.

Conclusions: The findings of the present study indicated that extroverted and introverted participants are likely to experience placebo analgesic effects and nocebo hyperalgesia effects, respectively. Both extroverted and introverted participants showed significant changes in brain networks under placebo response. Variation in emotional control and ventromedial prefrontal cortex inactivity may constitute the bulk of the personality differences in placebo analgesia. Differences in the regulation of the sensory conduction system (SCS) and release of the emotional circuit could be important factors affecting personality differences in nocebo hyperalgesia.

Keywords: Pain; functional brain imaging; personality; placebo effect; ventromedial prefrontal cortex

Submitted Jul 03, 2020. Accepted for publication Dec 21, 2020.

doi: $10.21037 /$ atm-20-5123

View this article at: http://dx.doi.org/10.21037/atm-20-5123

^ ORCID: 0000-0001-8225-8678. 


\section{Introduction}

The phenomena of placebo and nocebo effects have been widely implicated in the fields of psychology and neuroscience (1-3). Some studies suggested that the placebo response can cause analgesic effects or relieve pain, while the nocebo effect can aggravate pain and even result in hyperalgesia $(1,4)$. In clinical practice, both placebo and nocebo effects can impact the therapeutic effect, especially in relation to pain $(5,6)$. Studies have revealed that $30 \%$ of patients with pain experience relief after taking placebo (7). However, if patients are affected by adverse expectations, their condition deteriorates (8).

Most research on the neural mechanisms of placebo analgesia and nocebo hyperalgesia has been done in the psychology and neurobiology fields (9). Some scholars believe that the opioid receptor mediated analgesia system is a vital mechanism of placebo analgesia, and the dopamine circuit and reward system may also be important factors affecting placebo-mediated effects (10). The cholecystokinin (CCK) system is considered an important factor in the nocebo response (11). CCK-mediated anxiety is also associated with the effects of placebo and nocebo. Some researchers have noted that placebo and nocebo effects might be mediated by neurotransmitters such as cyclooxygenase and prostaglandin (12). However, the underlying neural mechanisms of placebo analgesia and nocebo hyperalgesia remain unclear.

Existing studies have not fully explored the complex brain networks of placebo and nocebo effects. With advancements in functional brain imaging technology, scholars have increasingly used this tool to assess brain neural networks, thereby providing comprehensive and macroscopic analyses of complex brain networks (13). At present, there are inconsistent findings from studies on the effects of placebo and nocebo. Some scholars suggest that placebo and nocebo effects are just two states of the same psychological reaction (14). The difference between two brain networks is attributed to their different activation statuses. Further, some researchers believe that there are two different brain networks underlying placebo and nocebo responses that are both intersecting and independent (15). However, because the opioid and CCK systems are distinct, they are inhibited by different antagonists, suggesting a certain degree of independence between the two networks. Therefore, determining the key factors associated with the placebo and nocebo effects will prove insight into understanding these neural mechanisms.
Personality factors may play a key role in placebo and nocebo effects (16). Accumulating evidence has revealed how anxiety impacts placebo analgesia and nocebo hyperalgesia. Studies have shown that having an introverted or depressed personality is more likely to cause anxiety, suggesting a close relationship between personality and the two effects (17). Similarly, extroverts have been reported as being more susceptible to verbal encouragement in pain management than introverts, which can induce a significant placebo effect and improves analgesia. Other researches have confirmed that extroverts have higher levels of activity in their prefrontal cortex, which may produce more of the reward effects of the dopamine release circuit (18). This suggests that the neural networks are different for different personalities. Brain network mechanisms of introverted and extroverted personalities are also proposed to be significantly different. Therefore, the distinction between the two personalities might be an important factor affecting placebo and nocebo responses. Based on these findings, we hypothesize that introverted and extroverted personalities correspond with significant differences in the neural signals underlying two effects. Assuming this is true, the brain mechanisms for placebo and nocebo effects can be identified.

In order to explore the state of connectivity among the brain areas, researchers began using Granger causality analysis (GCA) (19), an analytical method used for obtaining brain temporal relationship information from time-series data. For instance, Some studies have suggested that the multivariate GCA (mGCA) could be applied to the analysis of time-series functional magnetic resonance imaging (fMRI) data, and could examine real-time functional neural network dynamics of samples (20). Investigations are currently ongoing on the directed relationship of regions with using GCA, however, no studies have been conducted on placebo analgesia and nocebo hyperalgesia. Moreover, because GCA only evaluates directed networks in the brain, functional connectivity (FC) between networks cannot be explored through GCA, and the functional connection intensity map based on the key brain areas of the network can effectively be supplemented. As an important structure of the brain, the anterior cingulate cortex (ACC) plays an important role in the higher brain functions $(21,22)$. Some subareas of the ACC may be used in identifying pain perception components (21). ACC is also a key region in dopamine reward release circuit, and is vital in placebo response (23). Therefore, the ACC as the region of interest (ROI) is a crucial strategy for exploring the key nodes of 


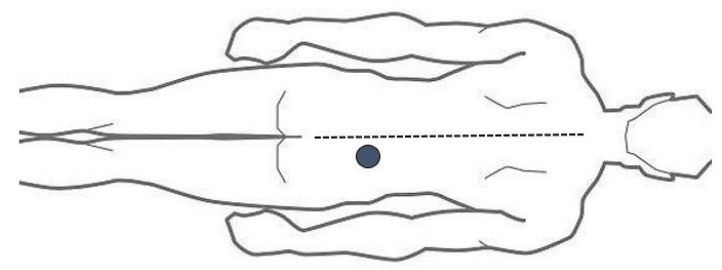

Figure 1 Location of the acute lower back pain model.

neural signals for placebo and nocebo responses.

In the present study, an acute lower back pain (ALBP) model was applied to discover the underlying brain network in placebo and nocebo effects (24). We manipulated participants' intervention expectations of the effectiveness of two patches, an analgesic patch (positive expectancy) and an algetic patch (negative expectancy). We then investigated the visual analogue scale (VAS) and changes in the neural signal before and after each intervention. GCA and FC analysis were used to explore the mechanisms for placebo and nocebo responses within an individual, and thus this study is unique among the other previously designed experiments. Using this method, we seek to develop an in-depth understanding of the mechanisms of placebo and nocebo responses, and to provide a bridge for further research.

We present the following article in accordance with the MDAR checklist (available at http://dx.doi.org/10.21037/ atm-20-5123).

\section{Methods}

\section{Participants}

Participants were recruited through an advertisement at Zhujiang Hospital. Participants were all from the southern region (Guangzhou, Guangdong Province, China), and all were right handed. Inclusion criteria were as follows: (I) candidate had not participated in prior psychological experiments; (II) candidate body mass index was within the standard $\pm 10 \%$; (III) candidate had not had psychiatric or medical conditions, including depression and mania in the previous 4 weeks; (IV) candidate did not have pain, including dysmenorrhea, and should not have been on medication, including antipyretics and sleeping pills in the previous 4 weeks; and $(\mathrm{V})$ candidates' scored $<50$ on a self-rating anxiety and depression scales (a score of $<50$ represents "normal"). Candidates were excluded if they had:
(I) organic brain disease; (II) history of craniocerebral injury; (III) drug dependence; (IV) severe neurological disorder; (V) metal component in body; (VI) claustrophobia; or (VII) had taken pain killers in the previous 4 weeks. All procedures performed in studies involving human participants were in accordance with the Declaration of Helsinki (as revised in 2013). The study protocol was approved by the Ethics Committee of Zhujiang Hospital affiliated to Southern Medical University (No. 2018-KFLLK-004) (25). All enrolled participants provided written informed consent before study commencement, and were debriefed at the end of the study. All participants were given the option to have their data excluded from the study if they had any concerns stemming from the necessary deception used in the experimental paradigm. However, no participant reported any concerns of this nature.

\section{Participant classification}

Participants were classified either as introverts or extroverts based on the Eysenck Personality Questionnaire (EPQ). Introverts were included in the introvert group (IG) and extroverts were included in the extrovert group (EG).

\section{Procedures}

We designed two patches, the analgesic patch (positive expectancy) and the algetic patch (negative expectancy). Both patches were similar to the analgesic patch commonly used in clinical practice.

The ALBP model was adopted from our previous study (24). According to the model, the point of injection was located $2 \mathrm{~cm}$ tangential of the spinous process of the fourth lumbar vertebra. An in-dwelling needle (24 gauge) was filled with sterile hypertonic saline $(10 \mathrm{~mL}, 5 \%)$ and attached via a long connecting tube to a computer-controlled power injector (Spectris Solaris EP; Medrad, Warrendale, PA, USA) before vertically inserting it into the above-described location at a depth of $1.5 \mathrm{~cm}$. After $1 \mathrm{~min}$, the hypertonic saline was intramuscularly injected from the computercontrolled power injector into the ALBP participant. This included a bolus injection $(0.1 \mathrm{~mL}$ within $5 \mathrm{~s})$ and subsequent continuous injection $(0.15 \mathrm{~mL} / \mathrm{min})$ to produce persistent ALBP (Figure 1).

\section{Training session}

Participants were familiarized with the ALBP scale and the visual analog scale (VAS), which they used to rate 


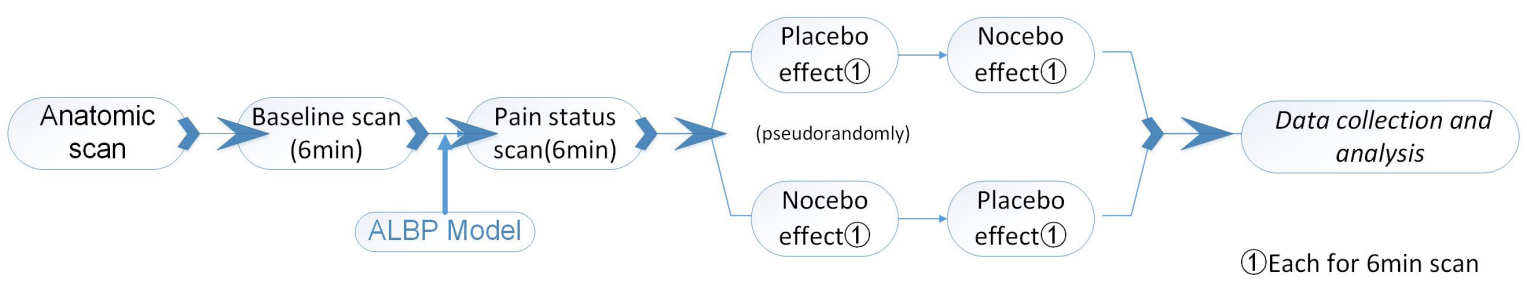

Figure 2 Experimental paradigm (scan session) for the participants.

their pain. Pain intensity was assessed on a 10-point VAS, with 0 indicating "no pain", and 10 indicating "worst pain imaginable". Pain level (i.e., distressing and intense) was measured with a 10 -point inhouse mood scale, with 0 indicating "infinitely small", and 10 indicating "excruciating" pain. Similarly, we recorded any discomfort of the participants to prevent any adverse reactions. VAS was collected at the end of each intervention.

\section{Behavioral conditioning session}

All of the participants were informed of the aim of the study, which was to investigate the analgesic effects of the analgesic patch and the algetic effects of the algetic patch on their experiences of pain. To improve the experimental environment for the participants, the process was conducted inside the magnetic resonance imaging (MRI) room. Participants were advised to apply one of the two patches (analgesic patch or algetic patch) to their right foot whenever they experienced ALBP. Participants would then start feeling pain alteration depending on the patch, with the order of patch application being random.

After the ALBP had stabilized, we proceeded with the experiment. In this conditioned paradigm, participants were informed that they would experience pain changes, depending on whether they had an analgesic patch or an algetic patch. They were instructed to focus on the captions displayed on a screen during this process. With an analgesic patch, the caption was "Please experience the effect of the analgesic patch", and for the algetic patch, the caption was "Please experience the effect of the algetic patch." At the end of each stimulation, we displayed the VAS on the screen, and the participants reported their pain scores. We reduced and increased the speed of hypertonic saline in uses of the analgesic patch and algetic patch, respectively. Thirty-four participants who distinguished between the pre- and post-intervention of the analgesic effects from analgesic patches or the algetic effects from algetic patches were selected to continue with the study.

\section{Scan session}

Participants were informed that the proceedings of the scan session would be similar to those of the previous session. This session was designed to assess the placebo and nocebo responses evoked by the expectancy induction in the previous session. With exception of the adjustment of hypertonic saline, other processes were similar during this process to previous sessions, that is, after ALBP establishment, the placebo and nocebo effects were induced, and the MRI data of different statuses were collected at the same time.

First, anatomical scans of the brain were obtained before the fMRI scans. Initially, participants were subjected to a baseline (normal) fMRI scan for 6 min. Next, the ALBP model was induced to every participant. After the ALBP had stabilized, the fMRI scan was used to evaluate the participant's pain status. This was followed by obtaining two fMRI scans for each ALBP participant (one scan during the placebo response and one scan during the nocebo response, pseudo-randomly), as the ALBP occurred continually throughout the scanning process.

To maximize the sustained effects caused by the previous intervention, we allowed for a 10-minute interval between the two scans. The main outcome of this experiment was the changes in VAS and fMRI signals caused by placebo and nocebo effects.

During scanning, participants were instructed to focus on the captions displayed on the screen. The caption for participants with an analgesic patch was "Please experience the effect of the analgesic patch, the scanning process is 6 min," while the caption for participants with an algetic patch was "Please experience the effect of the algetic patch, the scanning process is 6 min." After the administration of each stimulus, we displayed the VAS on the screen, and the participants reported their pain scores (Figure 2).

\section{Brain imaging}

The experiment was performed in the Department of 
Radiology of Zhujiang Hospital, China. Structural and functional scans were acquired using a 3.0 T Philips Achieva MRI System (Royal Philips Electronics, Eindhoven, the Netherlands) with an 8-channel head array coil equipped for echo-planar imaging (EPI). The images were axial and parallel to the anterior commissure-posterior commissure line, which covered the whole brain. Structural images were collected using a $T_{1}$-weighted fast spin-echo sequence (repetition time/echo time $=25 / 3 \mathrm{~ms}$, flip angle $=30^{\circ}$, matrix $=256 \times 256$, thickness $=5 \mathrm{~mm}$, slice $=24$, slice gap $=0.7 \mathrm{~mm}$ ) before functional imaging. Blood oxygenation leveldependent functional imaging was conducted using a $\mathrm{T}_{2}{ }^{*}$ weighted, single-shot, gradient-recalled EPI sequence (repetition time/echo time $=2,000 / 35 \mathrm{~ms}$, flip angle $=90^{\circ}$, matrix $=64 \times 64$, thickness $=5 \mathrm{~mm}$, slice $=24$, slice gap $=0.7 \mathrm{~mm}, \mathrm{NSA}=1,180$ time points for a total of $360 \mathrm{~s}$ ). Further, fMRI image collection was preceded by five dummy scans to minimize gradient distortion.

\section{Preprocessing of fMRI data}

The preprocessing analysis of fMRI image data was carried out in the Data Processing Assistant for Resting-State fMRI (DPARSF, http://www.restfmri.net) on MATLAB R2013b (Mathworks, Natick, CA, USA). Preprocessing analysis of Blood oxygen level-dependent (BOLD) time-series data included the following steps: (I) removal of the first 10 volumes from each BOLD time-series data to reduce the influence of magnetization inhomogeneities; (II) slice-time correction; (III) head movement correction (no participant data was excluded); (IV) spatial normalization to standard Montreal Neurological Institute (MNI) space; (V) spatial smoothing (6-mm full-width at half-maximum Gaussian kernel) to enhance signal-to-noise ratio (SNR) quality; (VI) detrend in the time-series data; and (VII) temporal filtering (0.01-0.08 Hz).

\section{Definition of seed region}

For consistency with the intramuscular part, data selection from the left ACC for the ROI $\left(3 \times 3 \times 3 \mathrm{~mm}^{3}\right)$ relied on the findings from a previous study of the anatomical location of the brain (26). MNI brain region coordinates were selected as the central voxel ROI $(x=-5, y=25, z=-10)$.

\section{ROI voxel FC analysis}

A function module (FC) of REST software (http://restfmri. net/forum/rest) was adopted.

This step was used to extract the individual time course of activity from the regions relative to the standard EPI space for the ACC. We obtained brain functionality images for each participant through Pearson's correlation coefficient analyses of the seed point and whole-brain voxel time series and standardization by Fisher's Z-transformation of correlation coefficients into $\mathrm{z}$ values.

A comparison of the placebo or nocebo status with a similar pain status group using false discovery rate (FDR) correction and setting the minimum voxel threshold of 50 revealed the key networks of the introverted and extroverted participants. These brain networks were associated with the placebo response of introverted participants, the nocebo response of introverted participants, the placebo response of extroverted participants, and the nocebo response of extroverted participants. The differential brain areas of each key network were included in the next analysis (Supplementary material).

\section{Whole-brain FC analysis (ROI-ROI analysis)}

Based on the statistical results, the brain areas of each key network in the introverted and extroverted personality groups were included in the respective ROI-ROI analysis. Brain functionality images for each participant were obtained through Pearson's correlation coefficient analyses of time series of each brain area and standardization using Fisher's $\mathrm{z}$-transformation of correlation coefficients into z-values.

\section{GCA analysis}

In this study, the REST-GCA module of REST was used for GCA analysis (http://restfmri.net/forum/rest).

\section{Bivariate GCA}

The bivariate GCA model was as follows:

$$
\begin{aligned}
& Y_{t}=\sum_{i=1}^{p} A_{i} X_{(t-i)}+\sum_{i=1}^{p} B_{i} Y_{(t-i)}+\ell_{t}+\varepsilon_{t} \\
& X_{t}=\sum_{i=1}^{p} A_{i}^{\prime} Y_{(t-i)}+\sum_{i=1}^{p} B_{i}^{\prime} X_{t-i}+C^{\prime} Z_{t}+\varepsilon_{t}^{\prime}
\end{aligned}
$$

In this bivariate GCA model (19), $A_{i}$ and $A_{i}$ are signedpath coefficients, $B_{i}$ and $B_{i}^{\prime}$ are autoregression coefficients, $\varepsilon_{t}$ and $\varepsilon_{t}$ are residual, $p$ is optimal lag parameter, and $Z_{t}$ is covariates. If the $A_{i}$ is significantly greater than or less than 0 , then the $X_{t}$ significantly Granger causes the $Y_{t}$. Moreover, if $A_{i}^{\prime}$ is significantly greater than or less than 0 , 


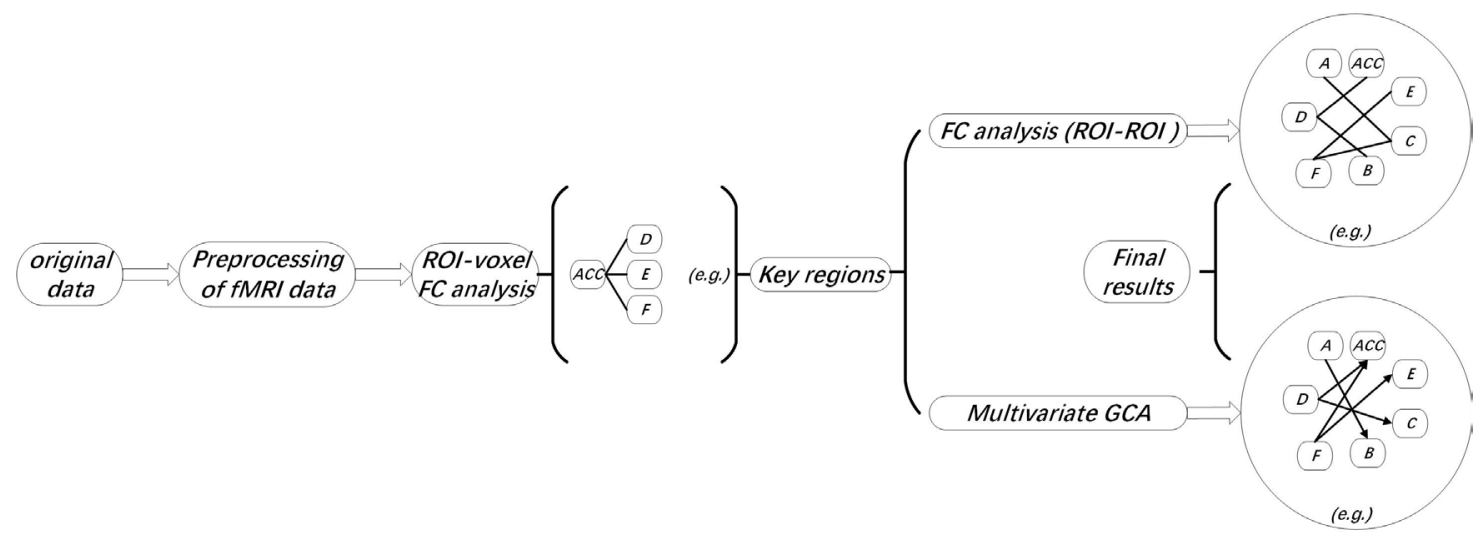

Figure 3 Flowchart of functional magnetic resonance imaging data analysis.

Table 1 Summary of pain scores and baseline characteristics of the 34 subjects

\begin{tabular}{lccc}
\hline & Extrovert & Introvert & P value \\
\hline No. & 19 & 15 & \\
Age & $25.05 \pm 3.31$ & $24.60 \pm 2.92$ & $>0.05$ \\
VAS (pain status) & $3.79 \pm 0.71$ & $4.33 \pm 0.62$ & $>0.05$ \\
VAS (placebo status) & $2.37 \pm 0.96^{\text {a\# }}$ & $3.40 \pm 0.63^{\text {a\# }}$ & $<0.05$ \\
VAS (nocebo status) & $4.47 \pm 0.70^{\text {b\# }}$ & $5.87 \pm 0.99^{\text {b\# }}$ & $<0.05$ \\
\hline
\end{tabular}

${ }^{a}$, placebo status vs. pain status; ${ }^{b}$, nocebo status vs. pain status; ", $\mathrm{P}<0.05$.

the $Y_{t}$ significantly Granger causes the $X_{t}(27)$.

\section{mGCA}

The mGCA model derived from the bivariate GCA model was as follows:

$$
\begin{aligned}
& Y_{1 t}=\sum_{i=1}^{p} A_{11}^{i} Y_{1}(t-i)+\ldots+\sum_{i=1}^{p} A_{1 n}^{i} Y_{n}(t-i)+C_{1} Z_{t}+\varepsilon_{t} \\
& \vdots \\
& Y_{n t}=\sum_{i=1}^{p} A_{n 1}^{i} Y_{1}(t-i)+\ldots+\sum_{i=1}^{p} A_{n n}^{i} Y_{n}(t-i)+C_{n} Z_{t}+\varepsilon_{t}
\end{aligned}
$$

Based on these statistical results, the brain areas of each key network in the introverted and extroverted personality groups were included in the respective mGCA analyses. These time-series data from the key network for each participant were incorporated into the mGCA. The mGCA coefficients obtained the strength and direction of the temporal correlations among the regions. Akaike Information Criterion (AIC) was applied to estimate lag parameter (28). We estimated the lag parameter was a lag of one-time resolution (TR) (2,000 ms) (27) (Figure 3).

\section{Statistical analysis}

We used the SPSS version 18.0 software (SPSS, Chicago, IL, USA) to calculate descriptive statistics (mean \pm standard deviation) for the VAS and other data. All statistical assessments were two-tailed. A P value $<0.05$ indicated a statistically significant result, which was consistent with the preliminary status of the trial.

The results of the ROI voxel analysis were calculated using two-tailed, two simple $t$-tests $(\mathrm{P}<0.05)$, and were corrected for multiple comparisons (FDR, $\mathrm{P}<0.05$ ).

We calculated the results of FC differences and GCA signed-path coefficient differences using two-tailed paired $t$-tests $(\mathrm{P}<0.05)$. At the subgroup level, the results were required to be statistically significant.

\section{Results}

Thirty-four healthy adults (15 introverts) aged 20 to 33 years completed the study. There were significant differences of VAS scores between placebo status and pain status in the IG, as well as between nocebo status and pain status $(\mathrm{P}<0.05)($ Table 1). Similarly, there were statistical differences of VAS scores between placebo status and pain status and between nocebo status and pain status in the EG $(\mathrm{P}<0.05)($ Table 1). Moreover, there were statistical differences of VAS scores between the introvert and EGs, in both placebo status and nocebo status $(\mathrm{P}<0.05$; Table 1). However, there were no statistical differences in the VAS scores regarding pain status $(\mathrm{P}>0.05$; Table 1$)$. 
Table 2 Changes of Functional connectivity network of two-status (Placebo-Pain) comparison in the introvert group

\begin{tabular}{|c|c|c|c|c|c|c|c|c|c|c|c|c|c|c|}
\hline & $\mathrm{ACC}$ & CPL & SMAI & PCC & BRS & TP & OFCr & OFCl & THS & PHP & DLPFCI & DLPFCr & SMAr & S2 \\
\hline ACC & N/A & -0.2891 & $-0.1943^{\star}$ & $0.3539^{\star}$ & $0.2887^{\star}$ & $-0.4041^{*}$ & $0.2082^{*}$ & -0.1927 & $-0.1977^{\star}$ & $-0.1854^{*}$ & $-0.2786^{\star}$ & $0.3974^{\star}$ & -0.2042 & $0.2686^{*}$ \\
\hline CPL & -0.2891 & N/A & -0.0326 & 0.0116 & 0.0524 & -0.1646 & -0.1220 & -0.0274 & -0.0487 & -0.1285 & -0.0666 & $0.1939^{*}$ & -0.0327 & 0.1032 \\
\hline SMAI & $-0.1943^{*}$ & -0.0326 & $\mathrm{~N} / \mathrm{A}$ & 0.0352 & 0.0578 & -0.0418 & -0.1079 & -0.0795 & 0.0894 & 0.0556 & $0.1862^{\star}$ & -0.0809 & -0.1394 & 0.0585 \\
\hline PCC & $0.3539^{*}$ & 0.0116 & 0.0352 & N/A & 0.0914 & 0.0279 & -0.0795 & 0.0179 & 0.0489 & 0.0071 & -0.0483 & 0.0082 & 0.1449 & -0.0270 \\
\hline BRS & $0.2887^{\star}$ & 0.0524 & 0.0578 & 0.0914 & $\mathrm{~N} / \mathrm{A}$ & -0.0083 & 0.0277 & 0.0336 & 0.0517 & -0.0467 & -0.0583 & 0.0339 & 0.0981 & -0.0034 \\
\hline TP & $-0.4041^{*}$ & -0.1646 & -0.0418 & 0.0279 & -0.0083 & N/A & 0.0415 & -0.0880 & -0.0426 & 0.0742 & -0.0169 & 0.0561 & -0.1408 & 0.0469 \\
\hline OFCr & $0.2082^{*}$ & -0.1220 & -0.1079 & -0.0795 & 0.0277 & 0.0415 & $\mathrm{~N} / \mathrm{A}$ & -0.0539 & -0.0188 & -0.0281 & -0.1041 & 0.1293 & -0.0720 & -0.0976 \\
\hline OFCl & -0.1927 & -0.0274 & -0.0795 & 0.0179 & 0.0336 & -0.0880 & -0.0539 & N/A & $-0.1594^{\star}$ & 0.0286 & -0.0850 & 0.0333 & 0.0331 & 0.1137 \\
\hline THS & $-0.1977^{*}$ & -0.0487 & 0.0894 & 0.0489 & 0.0517 & -0.0426 & -0.0188 & $-0.1594^{*}$ & N/A & 0.01907 & -0.0538 & -0.0305 & 0.0819 & 0.0748 \\
\hline PHP & $-0.1854^{*}$ & -0.1285 & 0.0556 & 0.0071 & -0.0467 & 0.0742 & -0.0281 & 0.0286 & 0.01907 & $\mathrm{~N} / \mathrm{A}$ & 0.0122 & -0.0513 & 0.0366 & -0.0447 \\
\hline DLPFCI & $-0.2786^{*}$ & -0.0666 & $0.1862^{\star}$ & -0.0483 & -0.0583 & -0.0169 & -0.1041 & -0.0850 & -0.0538 & 0.0122 & N/A & 0.0426 & -0.0818 & 0.0858 \\
\hline DLPFCr & $0.3974^{*}$ & $0.1939^{\star}$ & -0.0809 & 0.0082 & 0.0339 & 0.0561 & 0.1293 & 0.0333 & -0.0305 & -0.0513 & 0.0426 & $\mathrm{~N} / \mathrm{A}$ & -0.0237 & -0.0997 \\
\hline SMAr & -0.2042 & -0.0327 & -0.1394 & 0.1449 & 0.0981 & -0.1408 & -0.0720 & 0.0331 & 0.0819 & 0.0366 & -0.0818 & -0.0237 & N/A & 0.0858 \\
\hline $\mathrm{S} 2$ & $0.2686^{*}$ & 0.1032 & 0.0585 & -0.0270 & -0.0034 & 0.0469 & -0.0976 & 0.1137 & 0.0748 & -0.0447 & 0.0858 & -0.0997 & 0.0858 & N/A \\
\hline
\end{tabular}

The table values are group mean functional connectivity strength with prediction going from row to column. *, the group means are significantly different between the placebo response and the pain status, where a within-group effect was also present in the placebo response (two-tailed tests; all $\mathrm{P}<0.05$ ). ACC, anterior cingulate cortex; BRS, brainstem; CPL, cerebellum posterior lobe; DLPFC, dorsolateral prefrontal cortex; OFC, orbitofrontal cortex; PCC, posterior cingulate cortex; PHP, parahippocampal gyrus; SMA, supplementary motor area; S2, secondary somatosensory area; THS, thalamus; TP, temporal pole.

\section{Whole-brain FC network analysis results}

Brain response of placebo effect in the IG (ROI-ROI)

Based on the results of the ROI-voxel, we performed the whole-brain FC (ROI-ROI) analysis. Compared with the pain status, the placebo response showed that the ACC increased FC in the posterior cingulate cortex (PCC), brainstem (BRS), right orbitofrontal cortex (OFC), right dorsolateral prefrontal cortex (DLPFC), and the secondary somatosensory area (S2). Nonetheless, it decreased FC in the supplementary motor area (SMA), temporal pole (TP), thalamus (THS), parahippocampal gyrus (PHP), and the left DLPFC. cerebellum posterior lobe (CPL) had increased $\mathrm{FC}$ in the right DLPFC, whereas left SMA increased FC in the left DLPFC. In contrast, left OFC decreased FC in the THS (Table 2 and Figure 4).

\section{Brain response of placebo response in the EG (ROI-ROI)}

Based on the results of the ROI-voxel, we performed the whole-brain FC (ROI-ROI) analysis. Compared with the pain status, the placebo response showed that the ACC increased $\mathrm{FC}$ in the PHP, putamen (PUT), rolandic operculum (RO), OFC, hippocampus gyrus (HP), left insular cortex (IC), and the SMA. However, it decreased FC in the fusiform gyrus
(FG), PCC and the caudate (CAU). Similarly, FG decreased FC in the PHP and RO. OFC increased FC in the THS and the SMA, whereas it decreased FC in the left IC. Moreover, $\mathrm{HP}$ increased FC in the ventromedial prefrontal cortex (VMPFC) but decreased FC in the right IC. PCC decreased FC in the VMPFC (Table 3 and Figure 5).

Brain response of the nocebo effect in the IG (ROI-ROI) Based on the results of the ROI-voxel, we performed the whole-brain FC (ROI-ROI) analysis. Relative to the pain status, the nocebo response showed that the ACC increased FC in the amygdala (AMYG) and VMPFC but decreased $\mathrm{FC}$ in the FG, middle temporal lobe (MTL), superior temporal lobe (STL), pregenual anterior cingulate cortex (pgACC), DLPFC, and the primary somatosensory area (S1). Besides, Left CPL increased FC in the angular gyrus (AG), whereas it decreased FC in the S1. The right CPL decreased FC in the MTL; however, CAU increased FC in the pgACC. Additionally, the right VMPFC decreased FC in the pgACC (Table 4 and Figure 6).

Brain response of the nocebo effect in the EG (ROI-ROI) Based on the results of the ROI-voxel, the whole-brain FC (ROI-ROI) analysis was performed. Relative to the pain 


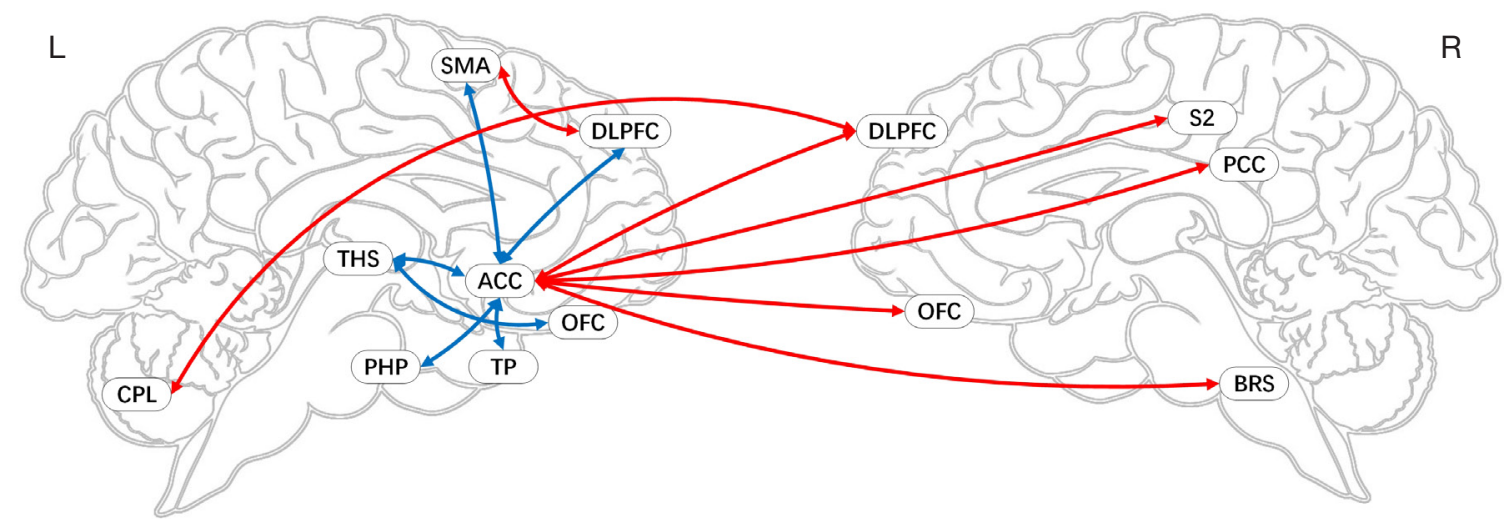

Figure 4 Functional connectivity (FC) network of a two-status (placebo-pain) comparison in the introvert group. Blue/red arrows present significantly greater decreased/increased FC in the placebo response when compared with the pain status. ACC, anterior cingulate cortex; BRS, brainstem; CPL, cerebellum posterior lobe; DLPFC, dorsolateral prefrontal cortex; OFC, orbitofrontal cortex; PCC, posterior cingulate cortex; PHP, parahippocampal gyrus; SMA, supplementary motor area; S2, secondary somatosensory area; THS, thalamus; TP, temporal pole.

Table 3 Changes of Functional connectivity network of two-status (Placebo-Pain) comparison in the extrovert group

\begin{tabular}{|c|c|c|c|c|c|c|c|c|c|c|c|c|c|c|c|}
\hline & ACC & PHP & $F G$ & CPL & PUT & THS & RO & OFC & $\mathrm{HP}$ & $\mathrm{ICl}$ & PCC & $\mathrm{ICr}$ & CAU & SMA & VMPFC \\
\hline $\mathrm{ACC}$ & $\mathrm{N} / \mathrm{A}$ & $0.2614^{*}$ & $-0.2985^{\star}$ & -0.1453 & $0.2610^{*}$ & 0.2004 & $0.2978^{\star}$ & $0.2611^{*}$ & $0.1524^{*}$ & -0.1797 & $-0.1541^{*}$ & $0.1863^{\star}$ & $-0.2105^{\star}$ & $0.2673^{\star}$ & 0.2384 \\
\hline PHP & $0.2614^{\star}$ & N/A & $-0.1748^{\star}$ & -0.0890 & -0.0063 & -0.0515 & 0.0522 & 0.0143 & -0.0746 & 0.0310 & 0.0734 & 0.0070 & -0.0058 & -0.0864 & 0.0224 \\
\hline $\mathrm{FG}$ & $-0.2985^{\star}$ & $-0.1748^{*}$ & N/A & 0.0718 & -0.0080 & -0.0052 & $-0.1661^{*}$ & -0.0826 & -0.0040 & -0.0324 & -0.0143 & -0.0952 & 0.1410 & -0.0038 & 0.0337 \\
\hline CPL & -0.1453 & -0.0890 & 0.0718 & N/A & -0.0651 & -0.0372 & -0.1432 & -0.0221 & 0.1022 & -0.0239 & 0.0617 & -0.0715 & -0.0894 & 0.0439 & 0.0126 \\
\hline PUT & $0.2610^{\star}$ & -0.0063 & -0.0080 & -0.0651 & $\mathrm{~N} / \mathrm{A}$ & 0.0722 & 0.0317 & 0.1815 & -0.0736 & -0.0169 & -0.1013 & -0.0294 & 0.0581 & -0.0415 & 0.0371 \\
\hline THS & 0.2004 & -0.0515 & -0.0052 & -0.0372 & 0.0722 & N/A & 0.0363 & $0.1070^{\star}$ & -0.0502 & -0.0744 & -0.0603 & -0.1575 & 0.0434 & 0.0470 & 0.0500 \\
\hline RO & $0.2978^{\star}$ & 0.0522 & $-0.1661^{*}$ & -0.1432 & 0.0317 & 0.0363 & N/A & 0.0729 & 0.0681 & 0.0149 & -0.0112 & -0.0072 & 0.0007 & -0.0708 & -0.0065 \\
\hline OFC & $0.2611^{\star}$ & 0.0143 & -0.0826 & -0.0221 & 0.1815 & $0.1070^{\star}$ & 0.0729 & N/A & 0.0587 & -0.0393 & 0.0304 & $-0.1857^{*}$ & 0.1177 & $0.1591^{*}$ & -0.0242 \\
\hline $\mathrm{HP}$ & $0.1524^{\star}$ & -0.0746 & -0.0040 & 0.1022 & -0.0736 & -0.0502 & 0.0681 & 0.0587 & N/A & 0.0860 & 0.0838 & $-0.2043^{*}$ & 0.0864 & 0.1005 & $0.1175^{\star}$ \\
\hline $\mathrm{ICl}$ & -0.1797 & 0.0310 & -0.0324 & -0.0239 & -0.0169 & -0.0744 & 0.0149 & -0.0393 & 0.0860 & N/A & 0.0092 & 0.0799 & -0.0810 & -0.0668 & -0.1017 \\
\hline PCC & $-0.1541^{*}$ & 0.0734 & -0.0143 & 0.0617 & -0.1013 & -0.0603 & -0.0112 & 0.0304 & 0.0838 & 0.0092 & N/A & -0.0412 & 0.0387 & 0.0335 & $-0.1648^{\star}$ \\
\hline $\mathrm{ICr}$ & $0.1863^{\star}$ & 0.0070 & -0.0952 & -0.0715 & -0.0294 & -0.1575 & -0.0072 & $-0.1857^{*}$ & $-0.2043^{\star}$ & 0.0799 & -0.0412 & $\mathrm{~N} / \mathrm{A}$ & 0.0069 & -0.0340 & 0.0154 \\
\hline $\mathrm{CAU}$ & $-0.2105^{\star}$ & -0.0058 & 0.1410 & -0.0894 & 0.0581 & 0.0434 & 0.0007 & 0.1177 & 0.0864 & -0.0810 & 0.0387 & 0.0069 & $\mathrm{~N} / \mathrm{A}$ & 0.2495 & -0.0780 \\
\hline SMA & $0.2673^{\star}$ & -0.0864 & -0.0038 & 0.0439 & -0.0415 & 0.0470 & -0.0708 & $0.1591^{\star}$ & 0.1005 & -0.0668 & 0.0335 & -0.0340 & 0.2495 & N/A & 0.0825 \\
\hline VMPFC & 0.2384 & 0.0224 & 0.0337 & 0.0126 & 0.0371 & 0.0500 & -0.0065 & -0.0242 & $0.1175^{\star}$ & -0.1017 & $-0.1648^{*}$ & 0.0154 & -0.0780 & 0.0825 & $\mathrm{~N} / \mathrm{A}$ \\
\hline
\end{tabular}

The table values are group mean functional connectivity strength with prediction going from row to column. *, the group means are significantly different between the placebo response and the pain status, where a within-group effect was also present in the placebo response (two-tailed tests; all $\mathrm{P}<0.05$ ). ACC, anterior cingulate cortex; CAU, caudate; CPL, cerebellum posterior lobe; FG, fusiform gyrus; HP, hippocampus gyrus; IC, insular; OFC, orbitofrontal cortex; PCC, posterior cingulate cortex; PHP, parahippocampal gyrus; PUT, putamen; RO, rolandic operculum; SMA, supplementary motor area; THS, thalamus; VMPFC, ventromedial prefrontal cortex. 


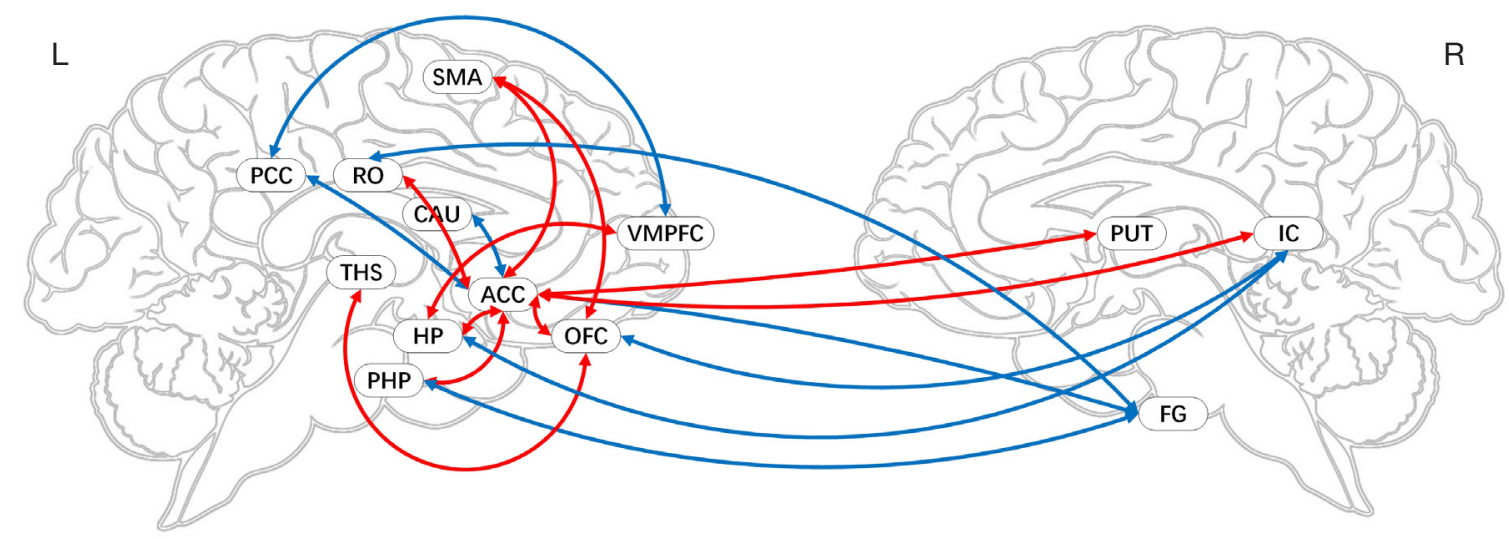

Figure 5 Functional connectivity (FC) network of a two-status (placebo-pain) comparison in the extrovert group. Blue/red arrows present significantly greater decreased/increased FC in the placebo response compared with the pain status. ACC, anterior cingulate cortex; CAU, caudate; CPL, cerebellum posterior lobe; FG, fusiform gyrus; HP, hippocampus gyrus; IC, insular; OFC, orbitofrontal cortex; PCC, posterior cingulate cortex; PHP, parahippocampal gyrus; PUT, putamen; RO, rolandic operculum; SMA, supplementary motor area; THS, thalamus; VMPFC, ventromedial prefrontal cortex.

Table 4 Changes of the Functional connectivity network of two-status (Nocebo-Pain) comparison in the introvert group

\begin{tabular}{|c|c|c|c|c|c|c|c|c|c|c|c|c|c|c|}
\hline & $\mathrm{ACC}$ & CPLI & CPLr & $\mathrm{FG}$ & $A G$ & AMYG & MTL & $\mathrm{CAU}$ & VMPFCr & STL & VMPFCl & pgACC & DLPFC & $\mathrm{S} 1$ \\
\hline ACC & N/A & -0.1959 & $-0.2290^{*}$ & $-0.3266^{*}$ & 0.2014 & $0.2262^{\star}$ & $-0.2893^{\star}$ & 0.1495 & $0.2303^{*}$ & $-0.3049^{*}$ & $0.3326^{\star}$ & $-0.2615^{\star}$ & $-0.2167^{*}$ & $-0.2350^{\star}$ \\
\hline CPLI & -0.1959 & $\mathrm{~N} / \mathrm{A}$ & 0.0777 & -0.2749 & $0.1302^{*}$ & -0.0192 & -0.2652 & 0.0311 & -0.0257 & -0.1237 & 0.0160 & -0.0531 & -0.1347 & $-0.3577^{*}$ \\
\hline CPLr & $-0.2290^{\star}$ & 0.0777 & N/A & -0.1775 & 0.1116 & -0.0702 & $-0.1294^{*}$ & -0.1165 & 0.0015 & 0.0153 & 0.0091 & -0.0903 & -0.2571 & -0.1270 \\
\hline $\mathrm{FG}$ & $-0.3266^{\star}$ & -0.2749 & -0.1775 & N/A & 0.1330 & -0.0045 & -0.1322 & 0.1152 & -0.1507 & -0.0950 & -0.0494 & -0.0107 & -0.0245 & -0.0298 \\
\hline$A G$ & 0.2014 & $0.1302^{*}$ & 0.1116 & 0.1330 & $\mathrm{~N} / \mathrm{A}$ & -0.0576 & 0.2298 & -0.0128 & 0.1325 & 0.1962 & 0.0095 & 0.0458 & 0.1517 & 0.0047 \\
\hline AMYG & $0.2262^{*}$ & -0.0192 & -0.0702 & -0.0045 & -0.0576 & N/A & -0.0092 & -0.1274 & -0.0097 & 0.0766 & -0.0382 & -0.0219 & 0.0355 & -0.0018 \\
\hline MTL & $-0.2893^{\star}$ & -0.2652 & $-0.1294^{\star}$ & -0.1322 & 0.2298 & -0.0092 & N/A & 0.1630 & -0.1009 & -0.1390 & -0.1385 & -0.0975 & 0.0691 & -0.1780 \\
\hline CAU & 0.1495 & 0.0311 & -0.1165 & 0.1152 & -0.0128 & -0.1274 & 0.1630 & N/A & -0.0608 & -0.0778 & -0.0112 & $0.1601^{\star}$ & 0.1300 & 0.0066 \\
\hline VMPFCr & $0.2303^{*}$ & -0.0257 & 0.0015 & -0.1507 & 0.1325 & -0.0097 & -0.1009 & -0.0608 & N/A & 0.0245 & 0.1073 & $-0.1136^{*}$ & -0.1056 & -0.1013 \\
\hline STL & $-0.3049^{\star}$ & -0.1237 & 0.0153 & -0.0950 & 0.1962 & 0.0766 & -0.1390 & -0.0778 & 0.0245 & $\mathrm{~N} / \mathrm{A}$ & -0.1026 & -0.1352 & -0.0848 & -0.0634 \\
\hline VMPFCI & $0.3326^{\star}$ & 0.0160 & 0.0091 & -0.0494 & 0.0095 & -0.0382 & -0.1385 & -0.0112 & 0.1073 & -0.1026 & $\mathrm{~N} / \mathrm{A}$ & -0.0037 & -0.0794 & -0.1155 \\
\hline pgACC & $-0.2615^{\star}$ & -0.0531 & -0.0903 & -0.0107 & 0.0458 & -0.0219 & -0.0975 & $0.1601^{*}$ & $-0.1136^{\star}$ & -0.1352 & -0.0037 & $N / A$ & 0.0936 & -0.1051 \\
\hline DLPFC & $-0.2167^{\star}$ & -0.1347 & -0.2571 & -0.0245 & 0.1517 & 0.0355 & 0.0691 & 0.1300 & -0.1056 & -0.0848 & -0.0794 & 0.0936 & $\mathrm{~N} / \mathrm{A}$ & -0.0545 \\
\hline S1 & $-0.2350^{*}$ & $-0.3577^{\star}$ & -0.1270 & -0.0298 & 0.0047 & -0.0018 & -0.1780 & 0.0066 & -0.1013 & -0.0634 & -0.1155 & -0.1051 & -0.0545 & N/A \\
\hline
\end{tabular}

The values presented in the table represent group mean functional connectivity strength with prediction going from row to column. ${ }^{*}$, the group means are significantly different between the nocebo response and the pain status, where a within-group effect was also present in the placebo response (two-tailed tests; all P<0.05). ACC, anterior cingulate cortex; AG, angular gyrus; AMYG, amygdala; CAU, caudate; CPL, cerebellum posterior lobe; DLPFC, dorsolateral prefrontal cortex; FG, fusiform gyrus; MTL, middle temporal lobe; pgACC, pregenual anterior cingulate cortex; STL, superior temporal lobe; S1, primary somatosensory area; VMPFC, ventromedial prefrontal cortex.

status, the nocebo response revealed that the ACC had increased FC in the OFC, AG, right MTL, DLPFC, and the S1. However, it decreased FC in the CPL, PHP, and the PCC. Moreover, CPL decreased FC in the S1. Similarly, the left PHP decreased FC in the OFC. The right PHP increased FC in the left MTL but decreased FC in the AG, right MTL, DLPFC, and the prefrontal cortex (PFC). Besides, AMYG increased FC in the pgACC, similar to 


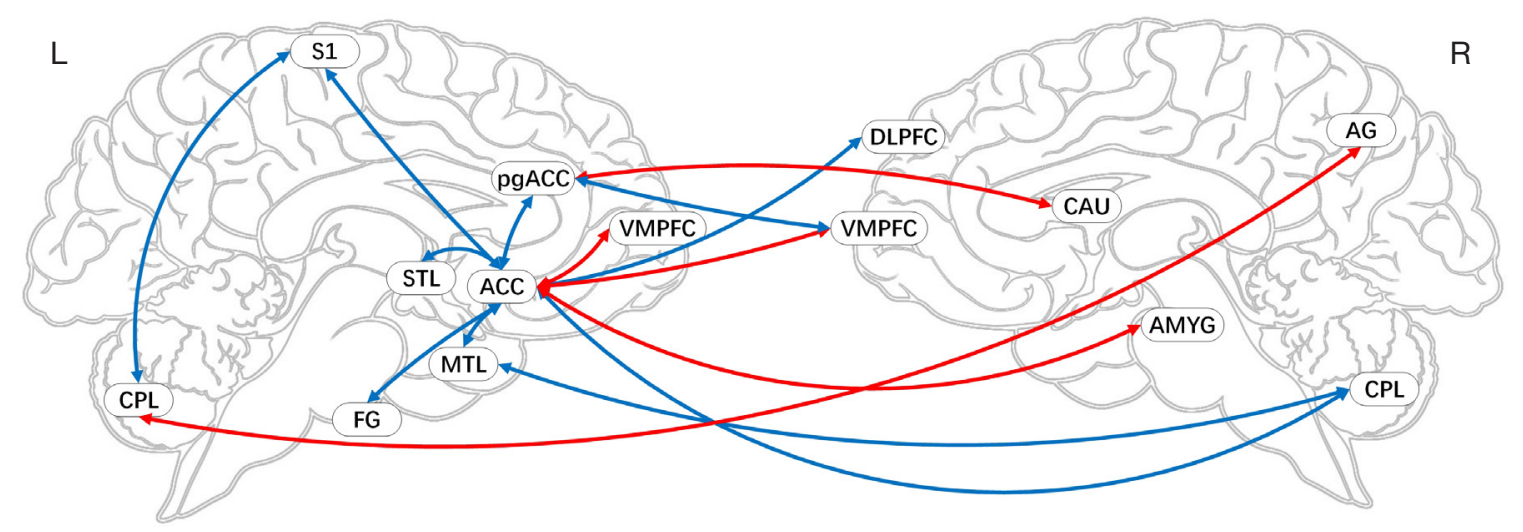

Figure 6 Functional connectivity (FC) network of two-status (nocebo-pain) comparison in the introvert group. Blue/red arrows present significantly greater decreased/increased FC in the nocebo response compared with the pain status. ACC, anterior cingulate cortex; AG, angular gyrus; AMYG, amygdala; CAU, caudate; CPL, cerebellum posterior lobe; DLPFC, dorsolateral prefrontal cortex; FG, fusiform gyrus; MTL, middle temporal lobe; pgACC, pregenual anterior cingulate cortex; STL, superior temporal lobe; S1, primary somatosensory area; VMPFC, ventromedial prefrontal cortex.

OFC in the AG and DLPFC (Table 5 and Figure 7).

\section{Granger causal network analysis results}

\section{Granger causal network of the placebo response in the} IG (mGCA)

Based on the results of the ROI-voxel, we performed the whole-brain mGCA. Compared with the pain status, the placebo response showed that the activity in the ACC predicted subsequent activation of the S2. Similarly, the activity in the left SMA predicted subsequent activation of the right $\mathrm{OFC}$. The activity in the BRS predicted subsequent activation of the right SMA. Moreover, the activity in the right OFC predicted the subsequent activation of the $\mathrm{S} 2$ and deactivation of the PHP. The activity in the left OFC and the THS predicted subsequent activation of the ACC and the S2, respectively. Additionally, the activity in the PHP predicted subsequent activation of the left DLPFC and deactivation of the right SMA. The activity in the right DLPFC and the $\mathrm{S} 2$ predicted subsequent activations of the TP and the right DLPFC, respectively. The activity in the right SMA predicted subsequent activation of the ACC and deactivation of the right DLPFC (Table 6 and Figure 8).

Granger causal network of the placebo response in the EG (mGCA)

Based on the results of the ROI-voxel, we performed the whole-brain mGCA. Relative to the pain status, the placebo response indicated that the activity in the ACC predicted subsequent activation of the THS. The activity in the CPL predicted subsequent activation of the PHP and deactivation of the left IC. However, the activity in the PUT predicted subsequent deactivation of the PHP, whereas the activity in the THS predicted subsequent activation of the HP. Moreover, the activity in the OFC predicted subsequent deactivation of the VMPFC, and the activity in the HP predicted subsequent activation of the PHP and deactivation of the CAU and VMPFC. The activity in the left IC predicted subsequent activation of the right IC and deactivation of the OFC. Additionally, the activity in the PCC predicted subsequent activation of the ACC and CAU. Similarly, the activity in the CAU predicted subsequent activation of the HP and the activity in the SMA predicted subsequent activation of the PHP and PCC (Table 7 and Figure 9).

\section{Granger causal network of the nocebo effect in the IG (mGCA)}

The whole-brain mGCA was performed based on the results of the ROI-voxel. Compared with the pain status, the nocebo response revealed that the activity in the ACC predicted subsequent activation of the right VMPFC, whereas the activity in the left CPL predicted subsequent deactivation of the right VMPFC. The activity in the right CPL predicted subsequent activation of the ACC. In contrast, the activity in the FG predicted subsequent deactivation of the AMYG and DLPFC. The activity in the AG predicted subsequent activation of the AMYG, whereas the activity in the CAU 
Table 5 Changes of Functional connectivity network of two-status (Nocebo-Pain) comparison in the extrovert group

\begin{tabular}{|c|c|c|c|c|c|c|c|c|c|c|c|c|c|c|c|}
\hline & ACC & CPL & PHPI & $\mathrm{PHPr}$ & AMYG & OFC & MTLI & $A G$ & MTLr & DLPFC & pgACC & PCC & PFC & MCC & $\mathrm{S} 1$ \\
\hline ACC & N/A & $-0.1868^{*}$ & $-0.2652^{*}$ & $-0.1544^{*}$ & 0.1379 & $0.3276^{*}$ & -0.1804 & $0.2347^{\star}$ & $0.2019^{\star}$ & $0.3599^{\star}$ & 0.1722 & $-0.2111^{\star}$ & 0.3089 & -0.1280 & $0.2474^{\star}$ \\
\hline CPL & $-0.1868^{*}$ & N/A & -0.1348 & 0.0546 & 0.0199 & -0.0530 & -0.0737 & -0.0019 & -0.0241 & -0.0318 & 0.0528 & 0.0080 & -0.0145 & -0.0142 & $-0.1598^{\star}$ \\
\hline PHPI & $-0.2652^{*}$ & -0.1348 & N/A & 0.0330 & -0.0094 & $-0.1286^{\star}$ & 0.0301 & 0.0098 & 0.0419 & -0.0564 & 0.0119 & 0.0713 & 0.1127 & -0.0614 & 0.0573 \\
\hline $\mathrm{PHPr}$ & $-0.1544^{*}$ & 0.0546 & 0.0330 & $\mathrm{~N} / \mathrm{A}$ & -0.0812 & 0.0064 & $0.2023^{*}$ & $-0.1440^{*}$ & $-0.2231^{*}$ & $-0.1562^{*}$ & -0.0539 & 0.0838 & $-0.1884^{*}$ & -0.0163 & 0.0484 \\
\hline AMYG & 0.1379 & 0.0199 & -0.0094 & -0.0812 & N/A & 0.1159 & -0.0551 & -0.0067 & -0.0928 & -0.1115 & $0.1367^{\star}$ & -0.0048 & 0.1068 & 0.0120 & 0.0206 \\
\hline OFC & $0.3276^{\star}$ & -0.0530 & $-0.1286^{\star}$ & 0.0064 & 0.1159 & N/A & -0.1483 & $0.1437^{\star}$ & 0.0538 & $0.2529^{*}$ & -0.0074 & -0.0003 & -0.0397 & -0.1007 & 0.0001 \\
\hline MTLI & -0.1804 & -0.0737 & 0.0301 & $0.2023^{\star}$ & -0.0551 & -0.1483 & $\mathrm{~N} / \mathrm{A}$ & 0.0263 & -0.0759 & 0.0056 & -0.1075 & -0.0370 & -0.0071 & -0.0638 & 0.0212 \\
\hline$A G$ & $0.2347^{\star}$ & -0.0019 & 0.0098 & $-0.1440^{\star}$ & -0.0067 & $0.1437^{\star}$ & 0.0263 & N/A & -0.0422 & 0.1118 & 0.0422 & 0.0130 & 0.0522 & -0.0127 & -0.0113 \\
\hline MTLr & $0.2019^{\star}$ & -0.0241 & 0.0419 & $-0.2231^{*}$ & -0.0928 & 0.0538 & -0.0759 & -0.0422 & $\mathrm{~N} / \mathrm{A}$ & -0.0118 & -0.0685 & 0.0341 & 0.1073 & 0.0085 & 0.1036 \\
\hline DLPFC & $0.3599^{\star}$ & -0.0318 & -0.0564 & $-0.1562^{*}$ & -0.1115 & $0.2529^{\star}$ & 0.0056 & 0.1118 & -0.0118 & $\mathrm{~N} / \mathrm{A}$ & 0.0485 & 0.0479 & -0.0969 & 0.0836 & -0.0247 \\
\hline pgACC & 0.1722 & 0.0528 & 0.0119 & -0.0539 & $0.1367^{\star}$ & -0.0074 & -0.1075 & 0.0422 & -0.0685 & 0.0485 & $\mathrm{~N} / \mathrm{A}$ & 0.0495 & -0.0209 & 0.0456 & 0.0048 \\
\hline PCC & $-0.2111^{*}$ & 0.0080 & 0.0713 & 0.0838 & -0.0048 & -0.0003 & -0.0370 & 0.0130 & 0.0341 & 0.0479 & 0.0495 & $\mathrm{~N} / \mathrm{A}$ & -0.1657 & -0.1533 & -0.1110 \\
\hline PFC & 0.3089 & -0.0145 & 0.1127 & $-0.1884^{*}$ & 0.1068 & -0.0397 & -0.0071 & 0.0522 & 0.1073 & -0.0969 & -0.0209 & -0.1657 & N/A & 0.0578 & -0.1136 \\
\hline MCC & -0.1280 & -0.0142 & -0.0614 & -0.0163 & 0.0120 & -0.1007 & -0.0638 & -0.0127 & 0.0085 & 0.0836 & 0.0456 & -0.1533 & 0.0578 & $\mathrm{~N} / \mathrm{A}$ & 0.0886 \\
\hline S1 & $0.2474^{*}$ & $-0.1598^{*}$ & 0.0573 & 0.0484 & 0.0206 & 0.0001 & 0.0212 & -0.0113 & 0.1036 & -0.0247 & 0.0048 & -0.1110 & -0.1136 & 0.0886 & N/A \\
\hline
\end{tabular}

The values presented in the table represent mean functional connectivity strength with prediction going from row to column. ${ }^{*}$ the group means are significantly different between the nocebo response and the pain status, where a within-group effect was also present in the placebo response (two-tailed tests; all P<0.05). ACC, anterior cingulate cortex; AG, angular gyrus; AMYG, amygdala; CPL, cerebellum posterior lobe; DLPFC, dorsolateral prefrontal cortex; MCC, mid-cingulate cortex; MTL, middle temporal lobe; OFC, orbitofrontal cortex; PFC, prefrontal cortex; pgACC, pregenual anterior cingulate cortex; PCC, posterior cingulate cortex; PHP, parahippocampal gyrus; S1, primary somatosensory area.

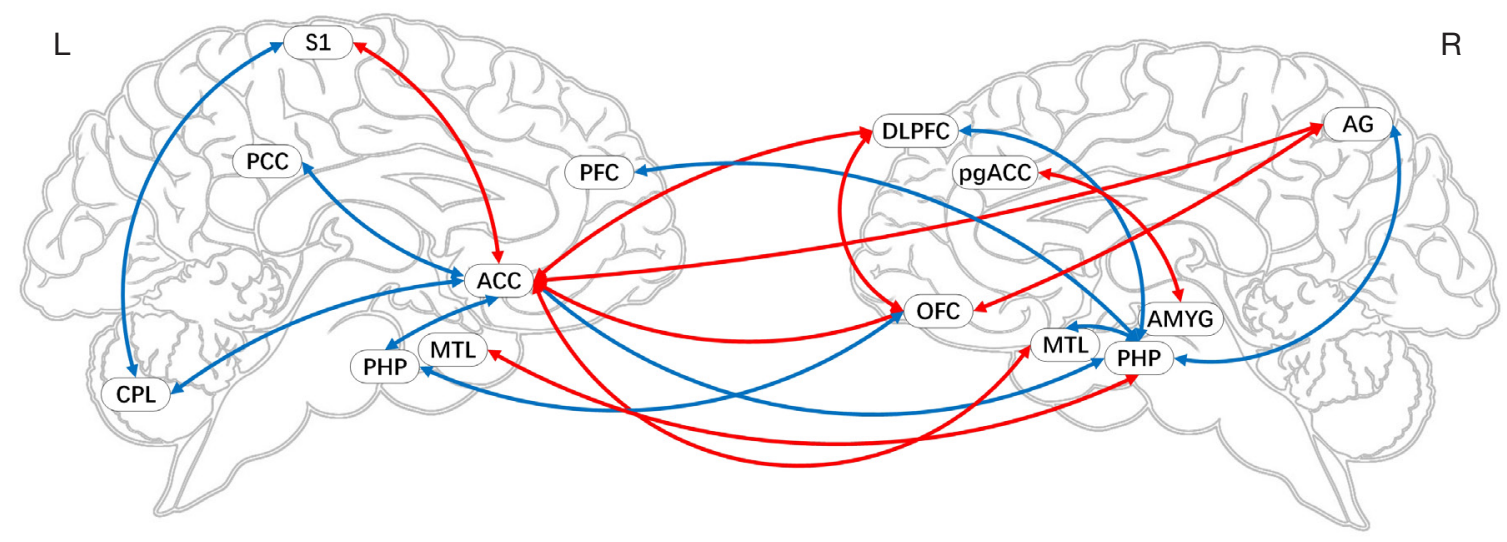

Figure 7 Functional connectivity (FC) network of a two-status (nocebo-pain) comparison in extroverts. Blue/red arrows present significantly greater decreased/increased FC in the nocebo response compared with the pain status. ACC, anterior cingulate cortex; AG, angular gyrus; AMYG, amygdala; CPL, cerebellum posterior lobe; DLPFC, dorsolateral prefrontal cortex; MCC, mid-cingulate cortex; MTL, middle temporal lobe; OFC, orbitofrontal cortex; PFC, prefrontal cortex; pgACC, pregenual anterior cingulate cortex; PCC, posterior cingulate cortex; PHP, parahippocampal gyrus; S1, primary somatosensory area. 
Table 6 Multivariate GCA results of the two-status (Placebo-Pain) comparison fit signed-path coefficients in the introvert group

\begin{tabular}{|c|c|c|c|c|c|c|c|c|c|c|c|c|c|c|}
\hline & ACC & CPL & SMAI & PCC & BRS & TP & OFCr & $\mathrm{OFCl}$ & THS & PHP & DLPFCI & DLPFCr & SMAr & S2 \\
\hline ACC & -0.0182 & 0.0787 & -0.0457 & 0.0195 & -0.0762 & 0.0158 & 0.0305 & 0.0142 & -0.0254 & -0.0523 & 0856 & -0.0638 & -0.1427 & $.2192^{*}$ \\
\hline CPL & -0.0716 & 0.0199 & 0.0000 & -0.0510 & -0.0420 & 0.0373 & 0.0230 & 0.0488 & 0.0857 & 0.0232 & -0.0454 & -0.0207 & 0.0521 & -0.1166 \\
\hline SMAI & 0.0165 & 0.0088 & -0.0414 & -0.0435 & 0.0172 & 0.0091 & $0.0515^{\star}$ & -0.0207 & 0.0270 & 0.0375 & 0.0260 & -0.0141 & 0.0232 & 0.0412 \\
\hline PCC & -0.0056 & -0.0299 & 0.0365 & -0.0132 & 0.0145 & 0.0106 & 0.0181 & 0.0409 & -0.0022 & 0.0228 & 0.0190 & 0.0220 & 0.0399 & -0.0131 \\
\hline OFCr & -0.0632 & 0.9885 & -0.1891 & -0.0849 & 0.0846 & 0.0049 & 0.0180 & 0.2210 & 0.1047 & $-0.1790^{*}$ & 0.1763 & -0.0204 & 0.1106 & $0.2544^{*}$ \\
\hline OFCl & $0.0881^{*}$ & 0.0523 & -0.0088 & -0.0545 & -0.0248 & 0.0029 & -0.0062 & -0.0217 & -0.0075 & -0.0296 & -0.0525 & -0.0250 & 0.0151 & -0.0140 \\
\hline THS & 0.0243 & 0.0393 & 0.0212 & -0.0114 & 0.0148 & -0.0040 & -0.0255 & 0.0050 & 0.0287 & -0.0485 & -0.0686 & -0.0136 & 0.0125 & $0.0708^{*}$ \\
\hline PHP & 0.0346 & 0.0191 & 0.0381 & -0.0439 & 0.0478 & -0.0132 & 0.0184 & 0.0350 & -0.0102 & -0.0116 & $0.1518^{*}$ & 0.0444 & $-0.0987^{\star}$ & 0.0776 \\
\hline SMAr & $0.0415^{\star}$ & 0.0305 & -0.0293 & -0.0756 & 0.0160 & 0.0147 & 0.0037 & -0.0237 & 0.0161 & 0.0137 & -0.0436 & $-0.0281^{*}$ & -0.0302 & -0.0020 \\
\hline S2 & -0.0268 & 0.0195 & 0.0065 & 0.0154 & -0.0146 & 0.0054 & -0.0010 & -0.0038 & -0.0197 & 0.0239 & -0.0230 & 0.0275 & 0.0491 & -0.0529 \\
\hline
\end{tabular}

The values presented in the table represent path coefficients with prediction going from row to column. *, the group means are significantly different between the placebo response and the pain status, where a within-group effect was also present in the placebo response (two-tailed tests; all $\mathrm{P}<0.05$ ). ACC, anterior cingulate cortex; BRS, brainstem; CPL, cerebellum posterior lobe; DLPFC, dorsolateral prefrontal cortex; OFC, orbitofrontal cortex; PCC, posterior cingulate cortex; PHP, parahippocampal gyrus; SMA, supplementary motor area; S2, secondary somatosensory area; THS, thalamus; TP, temporal pole.

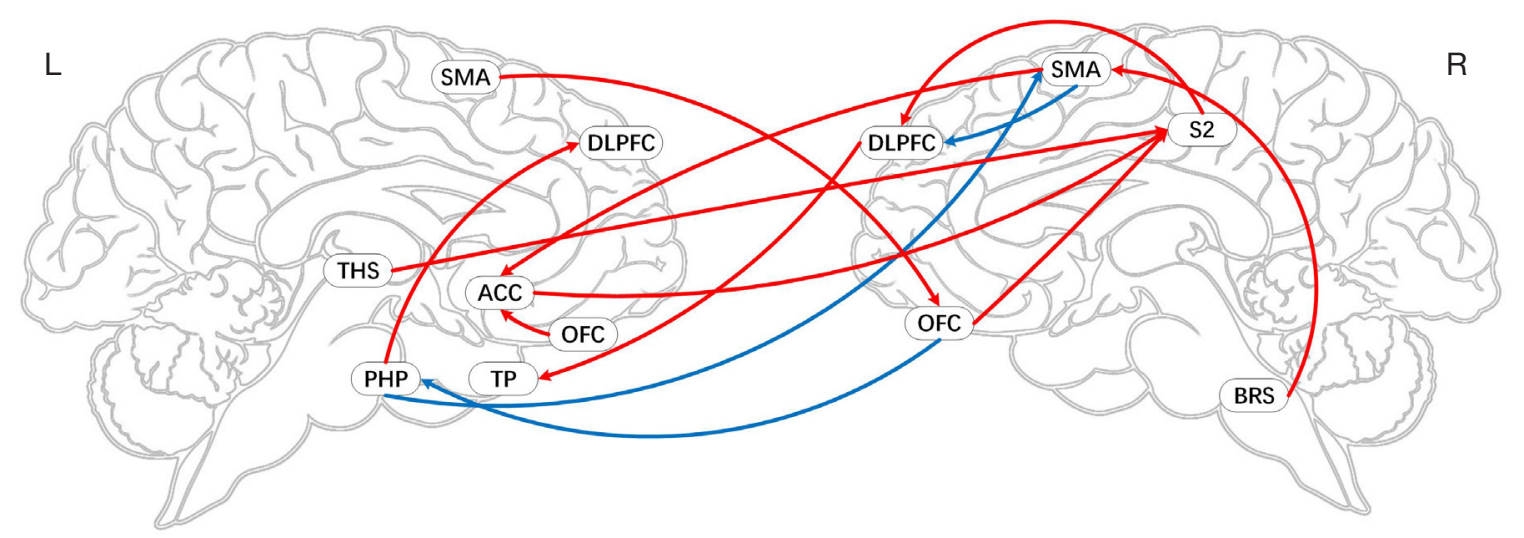

Figure 8 Brain map of a two-status (placebo-pain) comparison of signed-path coefficients from the multivariate Granger causality analysis in the introvert group. Blue/red arrows present significantly greater deactivation/activation of following region activity in the placebo response compared with the pain status. ACC, anterior cingulate cortex; BRS, brainstem; CPL, cerebellum posterior lobe; DLPFC, dorsolateral prefrontal cortex; OFC, orbitofrontal cortex; PCC, posterior cingulate cortex; PHP, parahippocampal gyrus; SMA, supplementary motor area; 2, secondary somatosensory area; THS, thalamus; TP, temporal pole.

predicted subsequent deactivation of the AG and S1. The activity in the left VMPFC predicted subsequent activation of the STL. Similarly, the activity in the pgACC predicted subsequent activation of the CAU. Moreover, the activity in the S1 predicted subsequent activation of the left VMPFC and deactivation of the AMYG (Table 8 and Figure 10).

\section{Granger causal network of the nocebo effect in the EG (mGCA)}

We performed the whole-brain mGCA based on the results 
Table 7 Multivariate GCA results of the two-status (Placebo-Pain) comparison fit signed-path coefficients in the extrovert group

\begin{tabular}{|c|c|c|c|c|c|c|c|c|c|c|c|c|c|c|c|}
\hline & ACC & HP & $G$ & PL & UT & THS & RO & DFC & HP & $\mathrm{ICl}$ & PCC & $\mathrm{ICr}$ & CAU & SMA & VMPFC \\
\hline ACC & & & & & & & & & & & & & & & \\
\hline PHP & & & & & & & & & 0.0 & 0.0 & & & & & \\
\hline$F G$ & 6 & -0.0 & & -0.0 & & & -0 & & & & & & & & \\
\hline CPL & -0.0 & 0.1 & & -0. & & 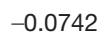 & 0.0 & & & & & & & & \\
\hline PU & -0.0025 & -0 . & & 7 & & 3 & & & & -0 & & & & & \\
\hline THS & 0.0951 & -0.0 & 0.0 & 0.0 & $-c$ & -0.0 & -0. & & 0.2 & 0.0629 & 0.0 & -0 . & 304 & & \\
\hline RO & -0.0106 & 0.0624 & -0.0084 & -0.0071 & -0.1063 & 0.0185 & -0.0032 & 0.0604 & -0.0110 & -0.1749 & 35 & 0.0216 & -0.0479 & 55 & 0.0384 \\
\hline OFC & 0.1550 & -0.0621 & -0.0479 & -0.0262 & -0.1037 & 0.0077 & -0.0540 & 0.0289 & 0.1102 & 0.0193 & 0.0836 & -0.0492 & -0.0951 & -0.1649 & $-0.0890^{\star}$ \\
\hline HP & 0.1226 & $0.0795^{\star}$ & -0.0284 & 0.0225 & 0.0584 & -0.0219 & -0.0035 & -0.0431 & 0.0117 & -0.1467 & 0.0124 & 0.0283 & $-0.1030^{\star}$ & -0.0752 & $-0.0607^{\star}$ \\
\hline $\mathrm{ICl}$ & 0.0159 & -0.0025 & 0.0188 & 0.0122 & -0.0069 & 0.0095 & 0.0309 & $-0.0464^{*}$ & 0.0396 & 0.0269 & -0.0016 & $0.0610^{\star}$ & 0.0340 & 0.0357 & -0.0084 \\
\hline PCC & $0.1304^{*}$ & 0.0524 & 0.0386 & 0.04 & -0 . & 30 & -0 & -0 . & 0.0525 & 0.0085 & -0 . & -0 . & $86^{\star}$ & 00 & 0.0218 \\
\hline $\mathrm{ICr}$ & -0.0059 & -0.0704 & -0.0008 & -0.0133 & -0.0472 & -0.0105 & -0.0357 & 0.0463 & 0.0169 & -0.0686 & 0.0263 & -0.0 & 401 & -0. & -0 \\
\hline CAU & 0.0220 & -0.0010 & -0.0367 & -0.0142 & -0.0802 & -0.0285 & 0.0127 & -0.0027 & $0.0838^{\star}$ & -0.0529 & -0.0009 & -0.0386 & 0.0279 & -0.0460 & 0.0170 \\
\hline SMA & -0.0059 & $0.0390^{\star}$ & 0.0030 & -0.0222 & 0.0859 & -0.0112 & -0.0304 & 0.0294 & 0.0219 & 0.0626 & $0.0482^{*}$ & -0.0225 & -0.0236 & -0.0624 & -0.0537 \\
\hline VMPFC & -0.0176 & 0.0511 & -0.0219 & 0.0610 & 0.0404 & 0.0363 & 0.0064 & 0.0743 & 0.0173 & 0.0460 & -0.0914 & -0.0401 & -0.0474 & 0.0178 & -0.0024 \\
\hline
\end{tabular}

The values presented in the table represent mean path coefficients with prediction going from row to column. ${ }^{*}$, the group means are significantly different between the placebo response and the pain status, where a within-group effect was also present in the placebo response (two-tailed tests; all $\mathrm{P}<0.05)$. ACC, anterior cingulate cortex; CAU, caudate; CPL, cerebellum posterior lobe; FG, fusiform gyrus; HP, hippocampus gyrus; IC, insular; OFC, orbitofrontal cortex; PCC, posterior cingulate cortex; PHP, parahippocampal gyrus; PUT, putamen; RO, rolandic operculum; SMA, supplementary motor area; THS, thalamus; VMPFC, ventromedial prefrontal cortex.

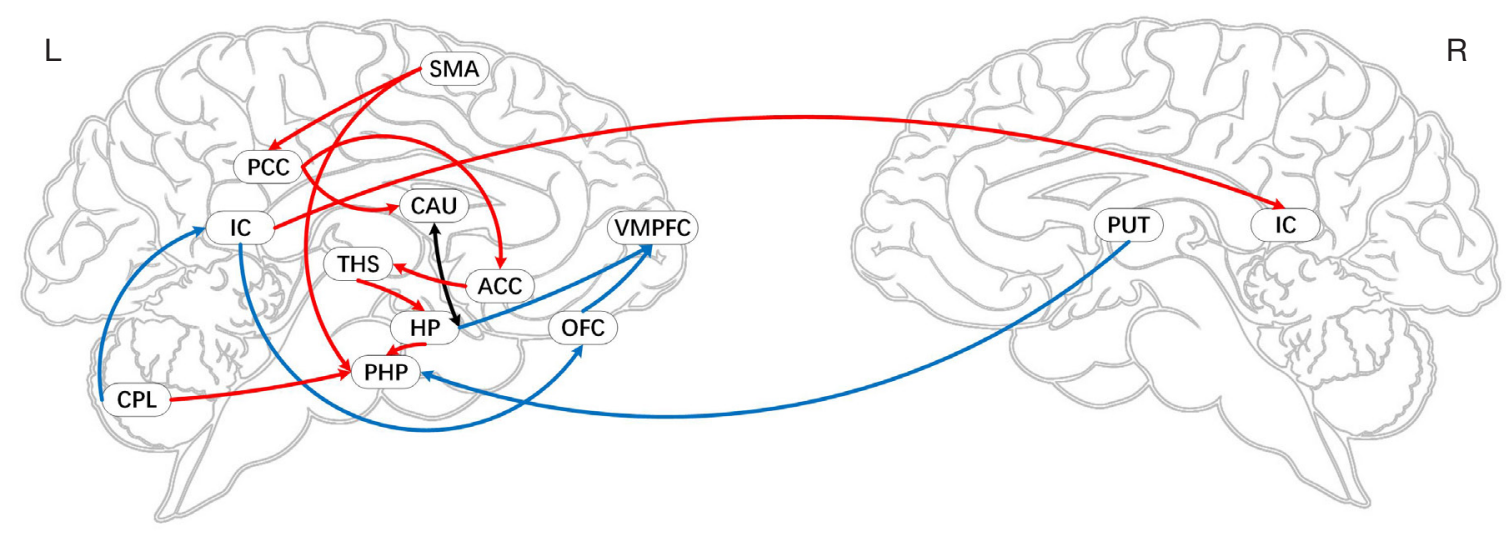

Figure 9 Brain map of a two-status (placebo-pain) comparison of signed-path coefficients from multivariate Granger causality analysis in the extrovert group. Blue/red arrows present significantly greater deactivation/activation of following region activity in the placebo response compared with the pain status. Black arrow presents a bidirectional prediction activity between the regions. ACC, anterior cingulate cortex; CAU, caudate; CPL, cerebellum posterior lobe; FG, fusiform gyrus; HP, hippocampus gyrus; IC, insular; OFC, orbitofrontal cortex; PCC, posterior cingulate cortex; PHP, parahippocampal gyrus; PUT, putamen; RO, rolandic operculum; SMA, supplementary motor area; THS, thalamus; VMPFC, ventromedial prefrontal cortex. 
Table 8 Multivariate GCA results of the two-status (Nocebo-Pain) comparison fit signed-path coefficients in the introvert group

\begin{tabular}{|c|c|c|c|c|c|c|c|c|c|c|c|c|c|c|}
\hline & $\mathrm{ACC}$ & CPLI & CPLr & $\mathrm{FG}$ & $A G$ & AMYG & MTL & $\mathrm{CAU}$ & VMPFCr & STL & VMPFCl & pgACC & DLPFC & $\mathrm{S} 1$ \\
\hline $\mathrm{ACC}$ & 0.0531 & 0.7804 & 908 & 0.0037 & 0.0852 & 3 & 32 & -0.0050 & $1827^{\star}$ & 0.0 & 09 & 0.0 & 0.0249 & 0.0712 \\
\hline CPLI & 0.0243 & 0.0277 & 0.0210 & -0.0190 & 0.0860 & -0.0411 & 0.0227 & 0.0658 & $-0.1069^{*}$ & -0.0583 & 0.0187 & -0.0481 & -0.0266 & 0.0326 \\
\hline CPLr & $0.1279^{\star}$ & 0.2104 & 0.0330 & 0.0439 & -0.0 & -0.0070 & -0.0 & 0.04 & -0.0 & 0.0460 & 0.0 & 0.0104 & -0.0720 & 0.0837 \\
\hline $\mathrm{FG}$ & 0.0138 & -0.8417 & 0.0555 & 0.0264 & 0.0090 & $-0.2042^{*}$ & 0.0895 & 0.0389 & 0.0803 & 0.0088 & 07 & 515 & $-0.1368^{*}$ & 0.1860 \\
\hline AG & 0.0278 & -0.1394 & 0.05 & 0.0454 & 0.0118 & $0.0852^{\star}$ & -0.0035 & -0.0 & 6 & 0.0 & -0.0164 & -0.0141 & 0.0578 & -0.0377 \\
\hline AMYG & -0.0218 & 0.0408 & -0.0 & 0.0003 & -0.0 & & 0.0029 & 0.0 & 242 & 0.0 & 0.0 & 45 & & 0.0600 \\
\hline MTL & -0.0554 & -0.8494 & 0.0288 & -0.0391 & -0.0221 & -0 & -0.0 & -0.0214 & & 0.0 & 6 & & 518 & 0.0 \\
\hline CAU & 0.0357 & -1.6797 & 316 & 0.0852 & $-0.1523^{*}$ & -0.0163 & 0.0852 & 0.0438 & -0.0781 & 0.2080 & -0.0176 & -0.2091 & -0.0512 & $-0.2865^{\star}$ \\
\hline VMPFCr & -0.0279 & -0.2866 & 0.0778 & -0.0303 & 0.0185 & 0.0382 & -0.0303 & -0.0484 & 0.0220 & 0.0436 & -0.0126 & 0.0966 & 0.0762 & -0.1074 \\
\hline STL & -0.0720 & -1.0749 & -0.0426 & 0.0112 & -0.0746 & 0.0169 & -0.1409 & 0.0283 & -0.0263 & -0.0353 & -0.0561 & -0.0364 & -0.0223 & 0.0453 \\
\hline VMPFCl & 0.1303 & -0.5451 & -0.0496 & 0.0296 & 0.1746 & -0.0332 & 0.0169 & 0.0411 & 0.0968 & $0.1449^{*}$ & 0.0066 & 0.1377 & 0.1829 & -0.2971 \\
\hline pgACC & -0.0228 & 0.6216 & -0.0171 & 0.0085 & 0.0451 & -0.0415 & -0.0493 & $0.0616^{\star}$ & 0.0054 & -0.0627 & 0.0116 & -0.0419 & 0.0034 & -0.0900 \\
\hline DLPFC & 0.0622 & 2.9775 & -0.0154 & 0.0590 & -0.0900 & -0.0190 & -0.0053 & -0.0032 & 0.0257 & 0.0408 & -0.0151 & 0.0400 & -0.0305 & 0.1025 \\
\hline S1 & -0.0324 & -0.2493 & -0.0041 & 0.0497 & -0.0230 & $-0.1404^{*}$ & -0.0063 & 0.0479 & -0.0431 & -0.0607 & $0.0532^{\star}$ & 0.0111 & -0.0186 & -0.0008 \\
\hline
\end{tabular}

The values presented in the table represent path coefficients with the prediction going from row to column. *, the group means are significantly different between the nocebo response and the pain status, where a within-group effect was also present in the nocebo response (two-tailed tests; all $\mathrm{P}<0.05$ ). ACC, anterior cingulate cortex; AG, angular gyrus; AMYG, amygdala; CAU, caudate; CPL, cerebellum posterior lobe; DLPFC, dorsolateral prefrontal cortex; FG, fusiform gyrus; pgACC, pregenual anterior cingulate cortex; MTL, middle temporal lobe; STL, superior temporal lobe; S1, primary somatosensory area; VMPFC, ventromedial prefrontal cortex.

of the ROI-voxel. Relative to the pain status, the nocebo response showed that the activity in the left PHP predicted subsequent deactivation of the left MTL. In contrast, the activity in the right PHP predicted subsequent activation of the PCC. Similarly, the activity in the AMYG predicted subsequent activation of the AG, PCC, and the PFC. The activity in the $\mathrm{OFC}$ predicted subsequent activation of the CPL and deactivation of the PFC. The activity in the AG predicted subsequent deactivation of the right MTL and the pgACC. Moreover, the activity in the right MTL and the DLPFC predicted subsequent activation of the AMYG and the AG, respectively. The activity in the pgACC predicted subsequent activation of the DLPFC, whereas the activity in the PCC predicted subsequent deactivation of the AMYG and mid-cingulate cortex (MCC). The activity in the PFC predicted subsequent deactivation of the CPL, left PHP, and the S1 (Table 9 and Figure 11).

\section{Discussion}

In the present study, we investigated the personality response differences of the placebo analgesia and nocebo hyperalgesia. The results indicated that there were significant differences in the pain scores between the introvert and EGs in both the placebo and nocebo responses. Moreover, the comparison results revealed that the EG had a more significant reduction in pain score in the placebo response, whereas the IG had a more significant increase in pain score in the nocebo response. This implies that extroverts are more prone to the analgesic effect of the placebo response, whereas introverts are more prone to the hyperalgesic effect of the nocebo response. We analyzed the brain networks of the two groups. The results revealed that both groups had decreased FC in the brain network and some feedback pathways in the placebo response. In contrast, the FC of the brain network for both groups increased in the nocebo response.

\section{Personality differences in the FC brain network for the placebo response}

In the present study, the IG showed a negative FC network centered on the ACC, including important brain areas of the pain-related network, such as the TP, OFC, THS, PHP, and the SMA. The EG showed a negative FC network centered on the ACC involving the FG, IC, PCC, CAU, 


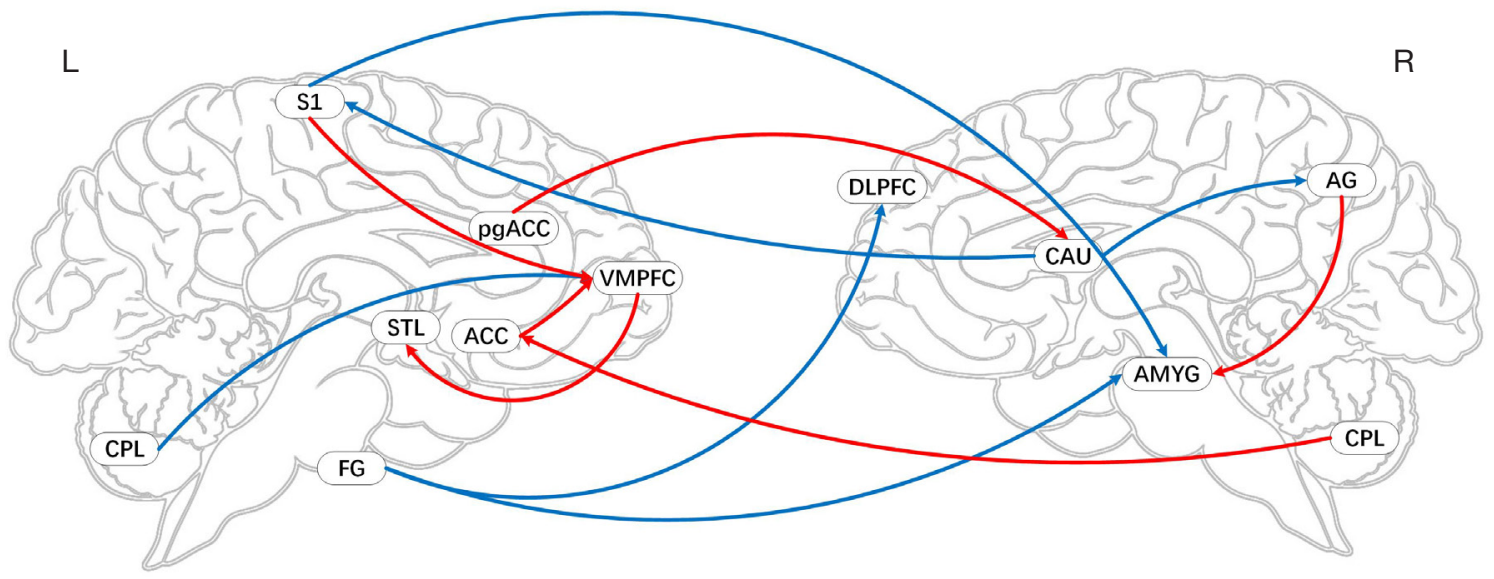

Figure 10 Brain map of the 2-status (nocebo-pain) comparison of the signed-path coefficients from multivariate Granger causality analysis in the introvert group. Blue/red arrows present significantly greater deactivation/activation of the following region activity in the nocebo response compared with the pain status. ACC, anterior cingulate cortex; AG, angular gyrus; AMYG, amygdala; CAU, caudate; CPL, cerebellum posterior lobe; DLPFC, dorsolateral prefrontal cortex; FG, fusiform gyrus; pgACC, pregenual anterior cingulate cortex; STL, superior temporal lobe; S1, primary somatosensory area; VMPFC, ventromedial prefrontal cortex.

Table 9 Multivariate GCA results of the two-status (Nocebo-Pain) comparison fit signed-path coefficients in the extrovert group

\begin{tabular}{|c|c|c|c|c|c|c|c|c|c|c|c|c|c|c|c|}
\hline & ACC & CPL & PHPI & $\mathrm{PHPr}$ & AMYG & OFC & MTLI & $A G$ & MTLr & DLPFC & pgACC & PCC & PFC & MCC & S1 \\
\hline ACC & 303 & 535 & 4 & 6 & 02 & 8 & 88 & -0 . & 0 & 46 & 74 & 0.0629 & 6 & 327 & 0.0 \\
\hline CPL & -0.0029 & 0.01 & 0.01 & 0.0293 & 0 & 69 & 8 & -0.0 & & -0.0266 & 0.0032 & 0.0548 & 0.0357 & 497 & -0 \\
\hline PHPI & 0.0093 & 0.01 & 0.0 & -0. & & 0.01 & -0 . & -0.0 & & 0.0349 & 0.0 & 0.0456 & 0.0109 & -0 & 0.0070 \\
\hline $\mathrm{PHPr}$ & -0.0019 & -0.0384 & -0.0212 & 0.0328 & 766 & -0.0327 & -0.0885 & -0.01 & 0.1008 & -0.0255 & 0.0150 & $0.1356^{*}$ & 0.0232 & -0.0017 & -0.0505 \\
\hline AMYG & -0.0289 & -0.0153 & 0.0284 & 0.0332 & 0.0271 & -0.0020 & 0.0653 & $0.0733^{\star}$ & -0.0602 & -0.0765 & 0.0143 & $0.0886^{\star}$ & $0.0693^{*}$ & 0.0397 & 0.0293 \\
\hline OFC & 0.0004 & $0.0831^{*}$ & -0.0170 & 0.0610 & -0.0048 & -0.0533 & -0.0313 & 0.0080 & -0.1351 & 0.0675 & 0.0325 & 0.0725 & $-0.0662^{*}$ & -0.0301 & 0.0580 \\
\hline MTLI & -0.0083 & -0.0084 & 0.0600 & -0.0017 & -0.0283 & 0.0076 & -0.0273 & -0.0110 & -0.0194 & 0.0209 & -0.0102 & -0.0155 & -0.0233 & -0.0309 & -0.0496 \\
\hline$A G$ & -0.0091 & -0.0015 & 0.0570 & 0.0890 & 030 & 0.0096 & 262 & 0.0384 & $-0.2488^{*}$ & -0.1371 & $-0.1728^{*}$ & -0.1088 & 0.0141 & -0.0044 & 0.0679 \\
\hline MTLr & -0.0120 & -0.0035 & -0.0030 & 0.0082 & $0.0654^{*}$ & 0.0217 & -0.0307 & -0.0055 & -0.0190 & 0.0053 & -0.0085 & 0.0021 & -0.0076 & 0.0097 & 0.0307 \\
\hline DLPFC & -0.0554 & 0.0084 & 0.0335 & 0.0061 & 0.0831 & -0.0646 & -0.0010 & $0.0612^{\star}$ & -0.0741 & -0.0560 & 0.0018 & -0.0193 & -0.0118 & -0.0139 & 0.0738 \\
\hline pgACC & -0.1078 & -0.0024 & -0.0524 & 0.0605 & 0.0267 & 0.0001 & 0.0499 & 0.0329 & -0.0446 & $0.1184^{*}$ & -0.0235 & 0.0045 & 0.0342 & 0.0314 & 0.0844 \\
\hline PCC & 0.0550 & -0.0583 & 0.0228 & -0.0313 & $-0.0808^{\star}$ & -0.0206 & 0.0117 & 0.0031 & -0.0544 & -0.0523 & -0.0043 & -0.0599 & -0.0388 & $-0.0834^{*}$ & 0.0185 \\
\hline PFC & -0.0894 & $-0.1673^{*}$ & $-0.1290^{*}$ & 0.0639 & 0.0094 & 0.0183 & 0.1139 & 0.0180 & -0.1549 & -0.0449 & -0.0061 & 0.0068 & -0.0182 & -0.0003 & $-0.1006^{\star}$ \\
\hline MCC & 0.0114 & -0.0220 & 0.0737 & -0.0282 & 0.0423 & 0.0384 & -0.0531 & -0.0098 & -0.0209 & -0.0008 & 0.0128 & -0.0216 & 0.0263 & -0.0247 & -0.0015 \\
\hline $\mathrm{S} 1$ & -0.0387 & -0.0453 & -0.0296 & -0.0139 & -0.0263 & 0.0444 & 0.0414 & 0.0000 & 0.0142 & -0.0175 & 0.0020 & 0.0796 & 0.0189 & -0.0122 & -0.0016 \\
\hline
\end{tabular}

The values presented in the table represent path coefficients with prediction going from row to column. *, the group means are significantly different between the nocebo response and the pain status, where a within-group effect was also present in the nocebo response (two-tailed tests; all $\mathrm{P}<0.05$ ). ACC, anterior cingulate cortex; AG, angular gyrus; AMYG, amygdala; CPL, cerebellum posterior lobe; DLPFC, dorsolateral prefrontal cortex; OFC, orbitofrontal cortex; PFC, prefrontal cortex; PCC, posterior cingulate cortex; pgACC: pregenual anterior cingulate cortex; PHP, parahippocampal gyrus; MCC, mid-cingulate cortex; MTL: middle temporal lobe; S1, primary somatosensory area. 


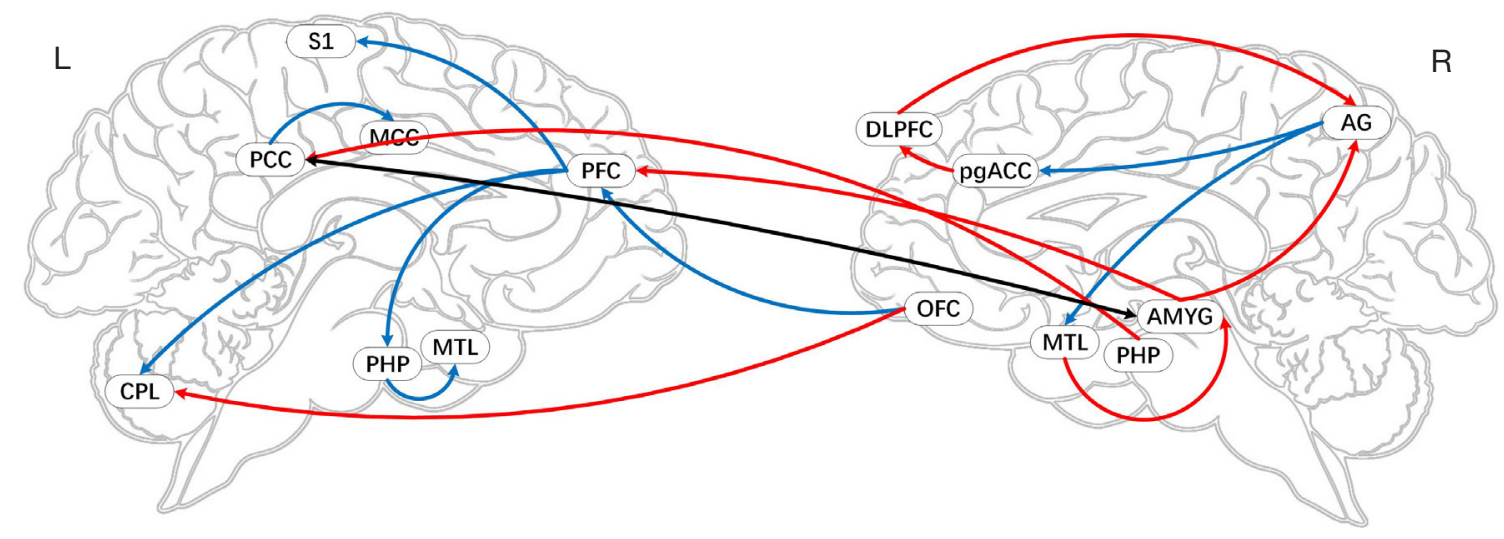

Figure 11 Brain map of the two-status (nocebo-pain) comparison of signed-path coefficients from multivariate Granger causality analysis in the extrovert group. Blue/red arrows present significantly greater deactivation/activation of the following region activity in the nocebo response compared with the pain status. Black arrow presents a bidirectional prediction activity between the regions. ACC, anterior cingulate cortex; AG, angular gyrus; AMYG, amygdala; CPL, cerebellum posterior lobe; DLPFC, dorsolateral prefrontal cortex; OFC, orbitofrontal cortex; PFC, prefrontal cortex; PCC, posterior cingulate cortex; pgACC, pregenual anterior cingulate cortex; PHP, parahippocampal gyrus; MCC, mid-cingulate cortex; MTL, middle temporal lobe; S1, primary somatosensory area.

and the PFC. The brain network composed of active brain regions in pain status is defined as the pain-related network $(29,30)$. Some brain regions of the pain-related network are involved in the production and transmission of pain. For example, IC and ACC form part of the emotional component (31). Decreased FC in these brain areas reduces pain-related network activity, thereby reducing the pain response (32). Compared with introverts, the pain-related network changes in extroverts are reflected in the decreased FC between the ACC and the multiple brain areas, as well as in the decreased FC among IC, VMPFC, and other brain areas $(29,33)$. These changes lead to a wider regulation of the FC network and more evident analgesic effects. This is consistent with our findings, where the pain scores in the EG were lower in the placebo response. Additionally, there is an increased FC network in extroverts, including the ACC, PUT, THS, HP, and the VMPFC. This helps to activate the endogenous opioid system $(34,35)$, releasing more opioids, and reducing the occurrence of a pain reaction (36). Notably, there was an increased FC between the ACC and BRS in our study, suggesting that introverts also experienced activation of the opioid system, but the activation area was not wider compared with that of extroverts. This could explain why the analgesic effect was significantly higher in extroverts relative to introverts. Both introverts and extroverts had increased FC between the OFC and ACC, suggesting that a placebo response could produce a more significant emotional regulation.
Negative emotions caused by a pain sensation produce a significant nocebo response $(37,38)$. The enhancement of emotional regulation effectively reduces the generation and transmission of negative emotions, improving the analgesic effect of the placebo response (39). There is evidence that DLPFC sends inhibitory signals to regulate painful sensory signals in the downstream signaling pathway under the effect of placebo (40). For the introverts in our study, the FC between the ACC and the bilateral DLPFC was the opposite, which reflected the characteristics of the placebo response of the pain sensory signaling pathway.

\section{Personality differences in the FC brain network for the nocebo response}

Brain areas, such as the AMYG, ACC, PFC, and the THS are closely associated with emotions $(41,42)$. The pain process also produces more negative emotions (43). In the present study, both the introverted and extroverted participants showed increased FC in these brain areas. The activation of these brain areas will accelerate the transmission and spread of negative emotions, aggravating the effect of anxiety on the nocebo effect. Moreover, studies suggest that the temporal lobe, cingulate cortex, and the PFC participate in emotional control and regulation $(44,45)$. Our findings indicate that the FC of These brain areas decreased in the two groups, and the emotional regulation network of introvert participants was more 
widely inactivated. This implies that negative emotions in the introvert subjects were more difficult to control and resulted in increased pain. Some studies have shown that activation of the CCK system is closely linked to the occurrence of anxiety, and anxiety is primarily thought to be an upstream pathway regulating the CCK system (46-48). The experience of negative emotions can activate the CCK system and plays a role in nocebo response. As a sensory conduction region in the brain, the CAU plays an important role in the nocebo effect (49). The results indicated that there is an increased FC between CAU and pgACC in introverts, suggesting that the nocebo effect increases information transfer to the pgACC through the CAU. In extroverts, there was increased FC between the DLPFC and the OFC. As an important brain area for information processing, activation of the $\mathrm{PFC}$ accelerates the rate of pain signal processing, reducing the effects of pain $(50,51)$. The introverts did not exhibit this change. Both introverts and extroverts showed increased FC between the AMYG and the ACC. As a key node of the emotional conduction pathway, the AMYG receives emotional signals from the THS and projects them to the PFC through the ACC (41). Changes in the brain network suggest that the brain network produces more emotional information in the nocebo response such as negative emotions (e.g., anxiety), which aggravates the pain response. Comparing the brain network between the placebo and nocebo responses in introverts and extroverts, we found that the placebo response was mainly mediated by the opioid receptor system in introverts, while the nocebo response was primarily caused by anxiety factors and the loss of emotional regulation, which additionally included activation of the CCK system. An overlap was seen in the two brain networks, but the subnetworks are quite different. In the extroverts, there was a difference in the emotional regulation pathway between the placebo and nocebo responses. The nocebo response tended to accelerate processing of prefrontal information, but this change was not found in the placebo group. This suggests that, although the brain networks for the placebo and nocebo responses overlap partially, differences still exist between them.

\section{Personality differences in the GCA brain network for the placebo response}

GCA results revealed that the regulation and feedback pathways in extroverted participants were mainly in the ipsilateral brain. In contrast, the regulatory pathways in the introverted participants overlapped in the bilateral brains, suggesting personality differences in the characteristics of the placebo response network. A reward system is a group of neural structures responsible for incentive salience, associative learning, and positive emotions, particularly involving pleasure as a core component $(52,53)$. The reward system includes the ventral striatum, PFC, ACC, IC, THS, and AMYG (54). A positive regulatory pathway appeared in the PCC, ACC, THS, HP, and the PHP in the EG. This suggests that the reward system was activated in the extroverted participants. The activation of the reward system produces increased amounts of neurotransmitters, such as dopamine and gamma-aminobutyric acid (GABA) (55), which promote a happy mood and reduce feelings of pain $(56,57)$. Changes in these brain networks also suggest that there could be activation of the opioid system to release analgesic substances. Moreover, these brain areas are closely associated with emotional regulation $(58,59)$, and their activation strengthens the emotional regulation of the placebo response. Although the positive regulation pathway between the ACC and the BRS was not found in the introverts, a positive regulation pathway between the BRS and SMA was observed, indicating the activation of the opioid system and suggesting that the opioid system could also be adjusted through the BRS-SMA-ACC pathway. Additionally, introverts showed positive regulation of the DLPFC-TP and OFC-ACC pathways. As vital brain areas for emotional control (60), TP and OFC activation encourages emotional control in introverts, suggesting a mechanism of emotional regulation under the placebo response. In extroverts, a positive regulation occurs between bilateral ICs, and there are abundant informationexchange nerve fibers between bilateral ICs (61). Right ICs are closely associated with mood stability and function by improving attention (62). Positive regulation of this pathway improves the body's alertness, maintaining more effective placebo analgesia. In extroverts, the VMPFC was negatively regulated by the OFC and HP. Previous studies have indicated that individuals with reduced VMPFC activity are more likely to be deceived and induced $(63,64)$. Extroverts were more likely to believe in the placebo effect, because of the decreased excitability of the VMPFC. In contrast, this pattern was not observed in introverts. There is a mutual feedback adjustment relationship between the CAU and HP. The CAU is an important structure of the dorsal striatum (65). It is thought to be related to the visual beauty response and also to the generation of love (66). HP is closely related 
to the occurrence of anxiety $(67,68)$. Feedback adjustment of these two brain areas reduces the occurrence of anxiety and increases the feeling of happiness, which plays a role in placebo analgesia.

\section{Personality differences in GCA brain network for the nocebo response}

The data presented here indicate that the regulatory signals were emitted from the OFC and transmitted through the $\mathrm{PFC}$ in extroverted participants, which had a negative impact on the S1, CPL, and PHP. This characterizes the brain network driving the nocebo response in extroverted participants while concurrently decreasing the regulation of emotion. As an essential emotional center, the AMYG receives emotional information from the THS and relays it into the PFC for analysis (69). A positive regulatory pathway of MTL-ACC-AMYG-PFC was identified in the brain network for the nocebo response in extroverted participants. The activation of this pathway indicates that multiple emotions are elicited under the nocebo response, as demonstrated in the present study. Elevated anxiety via the direct pathway or the CCK system participates in the execution of the nocebo effect (70). Of note, a significantly negative regulation was found among the ACC, pgACC, MCC, and PCC in extroverts (71). This highlights that the internal transmission of pain information is blocked in the cingulate cortex, and each brain area mutually inhibits the function of each sensory information processing system. For the extroverts, this represents the characteristics of the feedback regulation mechanism for the nocebo response. Moreover, the PFC had a negative regulation of the S1 in extroverts, while it had a negative regulation of the $\mathrm{CAU}$ in the introverts, indicating personality differences in the regulation of the sensory cortex for the nocebo response. It is worth noting that the brain network of extroverts primarily displayed an activated emotional release system (i.e., emotional circuit), suggesting the role of anxiety in the brain network for the nocebo response in extroverts. In contrast to extrovert subjects, introvert subjects primarily showed the regulation of the sensory conduction system (SCS). Analysis of brain networks for the placebo and nocebo responses in each subgroup revealed that the brain network for the placebo response was largely characterized by activation of opioid and reward systems accompanied by alterations in the emotional regulation system in the introvert subjects. For the nocebo response, the brain network primarily showed alterations in the sensory information transmission system and some emotional regulation systems. In comparison, the brain network for the placebo response in extroverts exhibited a broader regulatory network whereas that for the nocebo response predominantly involved the emotional release and emotional processing systems.

\section{Limitations}

While the results presented here clearly show personality differences in placebo analgesia and nocebo hyperalgesia, the present study has some important limitations. First, although we employed the ROI-voxel FC method to reveal the key brain areas driving the placebo and nocebo responses, some of the brain areas are not part of the network. Therefore, more seed points (e.g., AAL timecourse analysis) should be used to comprehensively explore the network in future research. Second, the study was performed in only young people, and therefore differences between participants of different ages were not examined. Third, the study only focused on the placebo/nocebo brain network in acute pain model participants, and did not investigate the more complex placebo/nocebo brain network in patients with acute or chronic pain, which is an area that enquires future research. Fourth, due to the limited number of participants, the present study lacks a multi-dimensional analysis of research data. Multi-dimensional analysis based on EPQ data, such as $\mathrm{N}$ (neuroticism) and $\mathrm{P}$ (psychoticism) factors, will be used in future research.

\section{Conclusions}

We find that that extroverts are more likely to produce placebo analgesic effects while introverts are more likely to produce nocebo hyperalgesia effects. Both extroverts and introverts exhibit significant changes in brain activity under placebo response. Emotional control and inactivity of the ventromedial prefrontal cortex might be the main reasons for personality differences in placebo analgesia. Notably, regulation of the SCS and release of the emotional circuit are also important factors contributing to personality differences in nocebo hyperalgesia.

\section{Acknowledgments}

We thank Yang JM from the Department of Neurology, Zhujiang Hospital, Southern Medical University in China for assistance. We thank all subjects for the assistance in the scanning. 
Funding: This work was supported by (I) National Natural Science Foundation of China (NNSFC), China; Contract grant number: 81772430; (II) Clinical Research Foundation of Southern Medical University, China; Contract grant number: LC2016PY037; (III) Basic and Applied Basic Research Foundation of Guangdong Province China; Contract grant number: 2019A1515110739; (IV) China Postdoctoral Science Foundation, China; Contract grant number: 2019M662995.

\section{Footnote}

Reporting Checklist: The authors have completed the MDAR checklist. Available at http://dx.doi.org/10.21037/atm-205123

Data Sharing Statement: Available at http://dx.doi. org/10.21037/atm-20-5123

Conflicts of Interest: All authors have completed the ICMJE uniform disclosure form (available at http://dx.doi. org/10.21037/atm-20-5123). The authors have no conflicts of interest to declare.

Ethical statement: The authors are accountable for all aspects of the work in ensuring that questions related to the accuracy or integrity of any part of the work are appropriately investigated and resolved. All procedures performed in studies involving human participants were in accordance with the Declaration of Helsinki (as revised in 2013). The study protocol was approved by the Ethics Committee of Zhujiang Hospital affiliated to Southern Medical University (No. 2018-KFLLK-004), with written informed consent obtained for all participants.

Open Access Statement: This is an Open Access article distributed in accordance with the Creative Commons Attribution-NonCommercial-NoDerivs 4.0 International License (CC BY-NC-ND 4.0), which permits the noncommercial replication and distribution of the article with the strict proviso that no changes or edits are made and the original work is properly cited (including links to both the formal publication through the relevant DOI and the license). See: https://creativecommons.org/licenses/by-nc-nd/4.0/.

\section{References}

1. Jensen KB, Kaptchuk TJ, Kirsch I, et al. Nonconscious activation of placebo and nocebo pain responses. Proc Natl Acad Sci U S A 2012;109:15959-64.

2. Benedetti $\mathrm{F}$. The opposite effects of the opiate antagonist naloxone and the cholecystokinin antagonist proglumide on placebo analgesia. Pain 1996;64:535-43.

3. Testa M, Rossettini G. Enhance placebo, avoid nocebo: How contextual factors affect physiotherapy outcomes. Man Ther 2016;24:65-74.

4. Benedetti F, Pollo A, Lopiano L, et al. Conscious expectation and unconscious conditioning in analgesic, motor, and hormonal placebo/nocebo responses. J Neurosci 2003;23:4315-23.

5. Kam-Hansen S, Jakubowski M, Kelley JM, et al. Altered placebo and drug labeling changes the outcome of episodic migraine attacks. Sci Transl Med 2014;6:218ra5.

6. Lesser H, Sharma U, LaMoreaux L, et al. Pregabalin relieves symptoms of painful diabetic neuropathy: a randomized controlled trial. Neurology 2004;63:2104-10.

7. Ernst E, Saradeth T, Resch K. The powerful placebo. Lancet 1991;337:611.

8. Drici MD, Raybaud F, De LC, et al. Influence of the behaviour pattern on the nocebo response of healthy volunteers. Br J Clin Pharmacol 1995;39:204-6.

9. Colloca L, Klinger R, Flor H, et al. Placebo analgesia: psychological and neurobiological mechanisms. Pain 2013;154:511-4.

10. Benedetti F, Durando J, Vighetti S. Nocebo and placebo modulation of hypobaric hypoxia headache involves the cyclooxygenase-prostaglandins pathway. Pain 2014;155:921-8.

11. Navratilova E, Xie JY, Meske D, et al. Endogenous opioid activity in the anterior cingulate cortex is required for relief of pain. J Neurosci 2015;35:7264-71.

12. Colloca L, Benedetti F. Placebo and nocebo effects: Unfolding the complex interplay between distinct phenotypes and physiological mechanisms. Psychology of Consciousness: Theory, Research, and Practice 2016;3:162.

13. Peciña M, Love T, Stohler CS, et al. Effects of the mu opioid receptor polymorphism (OPRM1 A118G) on pain regulation, placebo effects and associated personality trait measures. Neuropsychopharmacology 2015;40:957-65.

14. Scott DJ, Stohler CS, Egnatuk CM, et al. Placebo and nocebo effects are defined by opposite opioid and dopaminergic responses. Arch Gen Psychiatry 2008;65:220-31.

15. Peciña $M$, Zubieta J. Over a decade of neuroimaging studies of placebo analgesia in humans: what is next? Mol Psychiatry 2015;20:415. 


\section{Page 20 of 21}

16. Schienle A, Höfler C, Übel S, et al. Emotion-specific nocebo effects: an fMRI study. Brain Imaging Behav 2018;12:180-7.

17. Clark LA, Watson D, Mineka S. Temperament, personality, and the mood and anxiety disorders. J Abnorm Psychol 1994;103:103-16.

18. Smillie LD, Cooper AJ, Proitsi P, et al. Variation in DRD2 dopamine gene predicts extraverted personality. Neurosci Lett 2010;468:234-7.

19. Granger CWJ. Investigating causal relations by econometric models and cross-spectral methods. Econometrica 1969;37:424-38.

20. Deshpande G, Laconte S, James GA, et al. Multivariate Granger causality analysis of fMRI data. Hum Brain Mapp 2009;30:1361-73.

21. Wager TD, Atlas LY, Botvinick MM, et al. Pain in the ACC? Proc Natl Acad Sci U S A 2016;113:E2474.

22. Piedimonte A, Guerra G, Vighetti S, et al. Measuring expectation of pain: Contingent negative variation in placebo and nocebo effects. Eur J Pain 2017;21:874.

23. Koban L, Ruzic L, Wager TD. Chapter 10 - Brain Predictors of Individual Differences in Placebo Responding. Placebo and Pain, 2013:89-102.

24. Shi Y, Liu Z, Zhang S, et al. Brain network response to acupuncture stimuli in experimental acute low back pain: an fMRI study. Evid Based Complement Alternat Med 2015;2015:210120.

25. China ScotPsRo. Administrative regulations on medical Institution, 1994.

26. Margulies DS, Kelly AM, Uddin LQ, et al. Mapping the functional connectivity of anterior cingulate cortex. Neuroimage 2007;37:579-88.

27. Hamilton JP, Chen G, Thomason ME, et al. Investigating neural primacy in Major Depressive Disorder: multivariate Granger causality analysis of resting-state fMRI timeseries data. Mol Psychiatry 2011;16:763-72.

28. Seth AK. A MATLAB toolbox for Granger causal connectivity analysis. J Neurosci Methods 2010;186:262-73.

29. Stern J, Jeanmonod D, Sarnthein J. Persistent EEG overactivation in the cortical pain matrix of neurogenic pain patients. NeuroImage 2006;31:721-31.

30. Karshikoff B, Jensen KB, Ingvar M, et al. LPS increases pain sensitivity by decreased pain inhibition and increased insular activation. Brain Behav Immun 2015;49:e1.

31. Ingvar M. Pain and functional imaging. Philos Trans $R$ Soc Lond B Biol Sci 1999;354:1347-58.

32. Lee MJ, Park BY, Cho S, et al. Increased connectivity of pain matrix in chronic migraine: a resting-state functional
Shi et al. Personality differences in placebo and nocebo effects

MRI study. J Headache Pain 2019;20:29.

33. Frot M, Mauguière F. Dual representation of pain in the operculo-insular cortex in humans. Brain 2003;126:438-50.

34. Petrovic P, Kalso E, Petersson KM, et al. Placebo and opioid analgesia--imaging a shared neuronal network. Science 2002;295:1737-40.

35. Schrepf A, Harper DE, Harte SE, et al. Endogenous opioidergic dysregulation of pain in fibromyalgia: a PET and fMRI study. Pain 2016;157:2217-25.

36. Millan MJ, Stein C, Parsons C, et al. Multiple opioid systems and pain: new directions. Eur J Pharmacol 1990;183:i.

37. Flaten MA. Pain-related negative emotions and placebo analgesia. Handb Exp Pharmacol 2014;225:81.

38. Schienle A, Höfler C, S Ü, Wabnegger A. Emotionspecific nocebo effects: an fMRI study. Brain Imaging Behav 2018;12:180-7.

39. Zhang W, Guo J, Zhang J, et al. Neural mechanism of placebo effects and cognitive reappraisal in emotion regulation. Prog Neuropsychopharmacol Biol Psychiatry 2013;40:364-73.

40. Eippert F, Bingel U, Schoell ED, et al. Activation of the opioidergic descending pain control system underlies placebo analgesia. Neuron 2009;63:533-43.

41. Aupperle RL, Dharol T, Ravindran LN, et al. Pregabalin effects on neural response to emotional faces. Front Hum Neurosci 2012;6:42.

42. Zhang S, Tian S, Chattun MR, et al. A supplementary functional connectivity microstate attached to the default mode network in depression revealed by resting-state magnetoencephalography. Prog Neuropsychopharmacol Biol Psychiatry 2018;83:76-85.

43. Bussone G, Grazzi L, Panerai AE. Pain, Emotion, Headache. Headache 2012;52:98-101.

44. Tucker DM, Luu P, Pribram KH. Ann N Y Acad Sci 1995;769:213-39.

45. Eckstein M, Markett S, Kendrick KM, et al. Oxytocin differentially alters resting state functional connectivity between amygdala subregions and emotional control networks: inverse correlation with depressive traits. NeuroImage 2017;149:458-67.

46. Ravard S, Dourish CT. Cholecystokinin and anxiety. Trends Pharmacol Sci 1990;11:271-3.

47. Bradwejn J, Koszycki D. The cholecystokinin hypothesis of anxiety and panic disorder. Ann N Y Acad Sci 1994;713:273-82.

48. Carlino E, Piedimonte A, Benedetti F. Nature of the placebo and nocebo effect in relation to functional neurologic 
disorders. Handb Clin Neurol 2016;139:597-606.

49. Napadow V, Li A, Loggia ML, et al. The imagined itch: brain circuitry supporting nocebo-induced itch in atopic dermatitis patients. Allergy 2015;70:1485.

50. Lorenz J, Minoshima S, Casey KL. Keeping pain out of mind: the role of the dorsolateral prefrontal cortex in pain modulation. Brain 2003;126:1079-91.

51. Schweinhardt P, Seminowicz DA, Jaeger E, et al. The anatomy of the mesolimbic reward system: a link between personality and the placebo analgesic response. J Neurosci 2009;29:4882-7.

52. Berridge KC, Kringelbach ML. Pleasure systems in the brain. Neuron 2015;86:646-64.

53. Schultz $W$. Neuronal reward and decision signals: from theories to data. Physiol Rev 2015;95:853-951.

54. Richard JM, Castro DC, Difeliceantonio AG, et al. Mapping brain circuits of reward and motivation: in the footsteps of Ann Kelley. Neurosci Biobehav Rev 2013;37:1919-31.

55. Yager LM, Garcia AF, Wunsch AM, et al. The ins and outs of the striatum: role in drug addiction. Neuroscience 2015;301:529-41.

56. Levar N, van Leeuwen JMC, Denys D, et al. Divergent influences of anterior cingulate cortex GABA concentrations on the emotion circuitry. NeuroImage 2017;158:136-44.

57. Jasmin L, Wu MV, Ohara PT. GABA puts a stop to pain. Curr Drug Targets CNS Neurol Disord 2004;3:487-505.

58. Ochsner KN, Gross JJ. The cognitive control of emotion. Trends Cogn Sci 2005;9:242-9.

59. Colasanti A, Rabiner EA, Lingford-Hughes A, et al. Opioids and anxiety. J Psychopharmacol 2011;25:1415-33.

60. Takahashi K, Taguchi T, Tanaka S, et al. Painful muscle stimulation preferentially activates emotion-related brain regions compared to painful skin stimulation. Neurosci Res 2011;70:285-93.

61. Zubieta JK, Bueller JA, Jackson LR, et al. Placebo effects

Cite this article as: Shi Y, Wang Y, Zeng Y, Zhan H, Huang S, Cai G, Yang J, Wu W. Personality differences in brain network mechanisms for placebo analgesia and nocebo hyperalgesia in experimental pain: a functional magnetic resonance imaging study. Ann Transl Med 2021;9(5):371. doi: 10.21037/atm-205123 mediated by endogenous opioid activity on mu-opioid receptors. J Neurosci 2005;25:7754-62.

62. Eckert MA, Menon V, Walczak A, et al. At the heart of the ventral attention system: the right anterior insula. Hum Brain Mapp 2009;30:2530-41.

63. Christ SE, Van Essen DC, Watson JM, et al. The contributions of prefrontal cortex and executive control to deception: evidence from activation likelihood estimate meta-analyses. Cereb Cortex 2009;19:1557-66.

64. Koestner BP, Hedgcock W, Halfmann K, et al. The role of the ventromedial prefrontal cortex in purchase intent among older adults. Front Aging Neurosci 2016;8:189.

65. Royce GJ, Laine EJ. Efferent connections of the caudate nucleus, including cortical projections of the striatum and other basal ganglia: An autoradiographic and horseradish peroxidase investigation in the cat. J Comp Neurol 1984;226:28-49.

66. Goldin PR. The neural bases of amusement and sadness: a comparison of block contrast and subject-specific emotion intensity regression approaches. NeuroImage 2005;27:26-36.

67. Walf AA, Frye CA. A review and update of mechanisms of estrogen in the hippocampus and amygdala for anxiety and depression behavior. Neuropsychopharmacology 2006;31:1097-111.

68. Barkus C, Mchugh SB, Sprengel R, et al. Hippocampal NMDA receptors and anxiety: At the interface between cognition and emotion. Eur J Pharmacol 2010;626:49-56.

69. Davis $M$, Whalen PJ. The amygdala: vigilance and emotion. Mol Psychiatry 2001;6:13-34.

70. Benedetti F, Amanzio M, Vighetti S, et al. The biochemical and neuroendocrine bases of the hyperalgesic nocebo effect. J Neurosci 2006;26:12014-22.

71. Hutchison WD, Davis KD, Lozano AM, et al. Painrelated neurons in the human cingulate cortex. Nat Neurosci 1999;2:403-5. 


\section{Supplementary}

Brain response of placebo response in introvert subjects (ROI-voxel)

In the FC network of the ACC, the findings indicated that compared with pain status, placebo status exhibited increased FC in the posterior cingulate cortex (PCC), brainstem (BRS), right orbitofrontal cortex (OFC), right dorsolateral prefrontal cortex (DLPFC), and the secondary somatosensory area (S2). Besides, the ACC exhibited significantly decreased FC in the cerebellum posterior lobe (CPL), supplementary motor area (SMA), temporal pole (TP), left OFC, thalamus (THS), parahippocampal gyrus (PHP), left DLPFC (Table S1 \& Figure S1).

Table S1 The locations of the brain areas showing significantly FC with the ACC in placebo response in introvert subjects. $(\mathrm{P}<0.05, \mathrm{FDR}<0.05)$

\begin{tabular}{|c|c|c|c|c|c|c|}
\hline \multirow{2}{*}{ Brain region } & \multirow{2}{*}{$R / L$} & \multicolumn{3}{|c|}{$\mathrm{MNI}$} & \multirow{2}{*}{ Voxel } & \multirow{2}{*}{ Z-score } \\
\hline & & $x$ & $Y$ & Z & & \\
\hline CPL & $\mathrm{L}$ & -12 & -36 & -51 & 76 & -5.4085 \\
\hline BRS & $\mathrm{R}$ & 18 & -39 & -33 & 200 & 6.6478 \\
\hline DLPFC & $\mathrm{L}$ & -33 & 36 & 6 & 129 & -6.0736 \\
\hline DLPFC & $\mathrm{R}$ & 21 & 66 & 6 & 60 & 6.08 \\
\hline OFC & $\mathrm{R}$ & 45 & 45 & -15 & 114 & 7.0473 \\
\hline OFC & $\mathrm{L}$ & -21 & 15 & -9 & 88 & -4.6067 \\
\hline PCC & $\mathrm{R}$ & 24 & -45 & 27 & 3467 & 12.6427 \\
\hline TP & $\mathrm{L}$ & -45 & 21 & -15 & 129 & -7.2788 \\
\hline THS & $\mathrm{L}$ & -15 & -24 & -3 & 64 & -6.5076 \\
\hline PHP & $\mathrm{L}$ & -27 & -33 & 3 & 51 & -6.099 \\
\hline S2 & $\mathrm{R}$ & 21 & -51 & 51 & 200 & 10.0914 \\
\hline SMA & $\mathrm{L}$ & -27 & -9 & 57 & 3287 & -11.1188 \\
\hline SMA & $\mathrm{R}$ & 9 & -12 & 60 & 59 & -5.3913 \\
\hline
\end{tabular}

FDR, false discovery rate; MNI, Montreal Neurological Institute. ACC, anterior cingulate cortex; BRS, brainstem; CPL, cerebellum posterior lobe; DLPFC, dorsolateral prefrontal cortex; OFC, orbitofrontal cortex; PCC, posterior cingulate cortex; PHP, parahippocampal gyrus; SMA, supplementary motor area; S2, secondary somatosensory area; THS, thalamus; TP, temporal pole. 

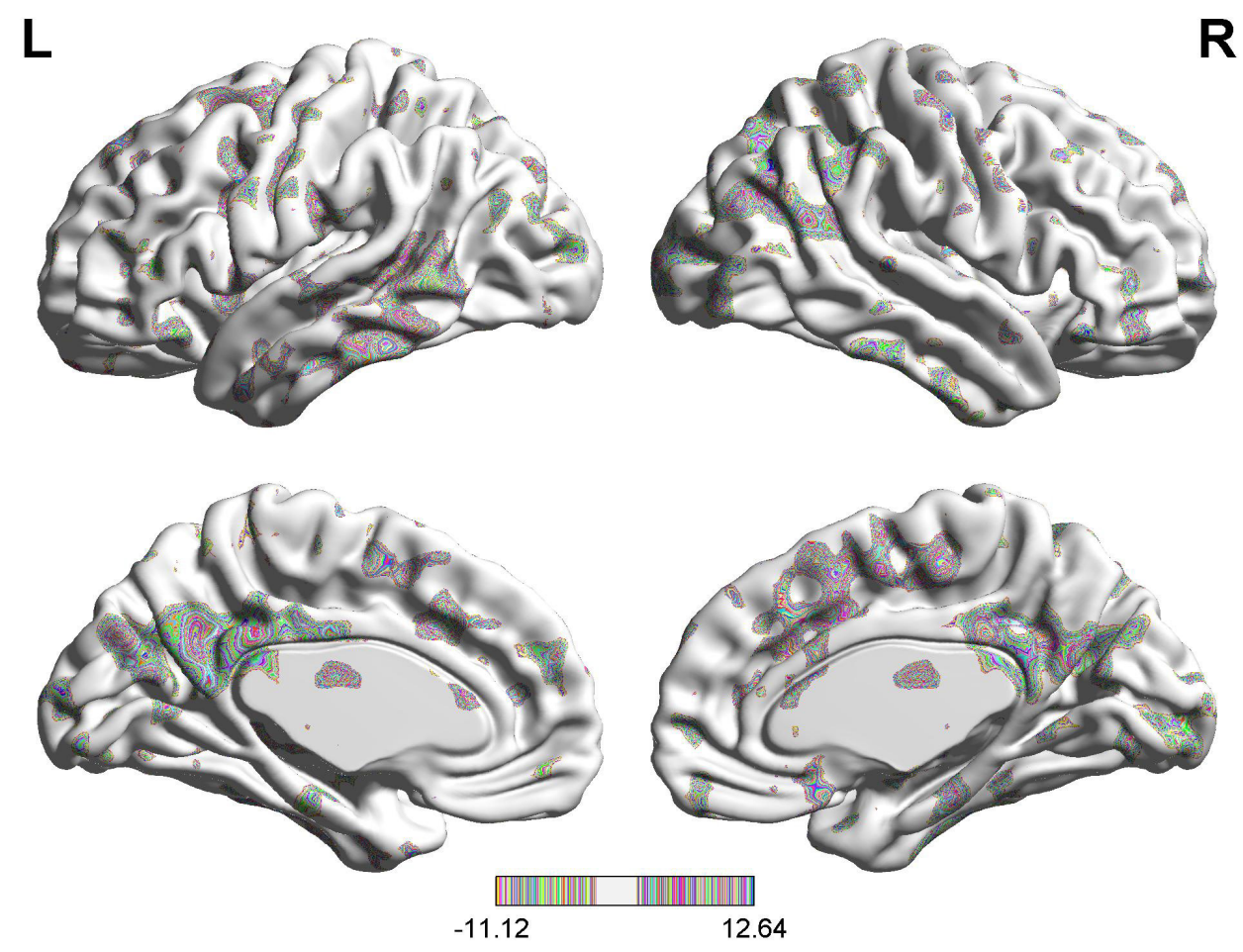

Figure S1 Brain areas showing significantly FC with the ACC in the placebo status in introvert group. 


\section{Brain response of placebo response in extrovert subjects (ROI-voxel)}

In the FC network of the ACC, the findings showed that compared with pain status, placebo status displayed increased FC in the PHP, putamen (PUT), THS, rolandic operculum (RO), OFC, hippocampus gyrus (HP), right insular cortex (IC), SMA, and the ventromedial prefrontal cortex (VMPFC). However, the ACC exhibited significantly decreased FC in the fusiform gyrus (FG), CPL, left IC, PCC, and the caudate (CAU) (Table S2 \& Figure S2).

Table S2 The locations of the brain areas showing significantly FC with the ACC in placebo response in extrovert subjects. $(\mathrm{P}<0.05, \mathrm{FDR}<0.05)$

\begin{tabular}{|c|c|c|c|c|c|c|}
\hline \multirow{2}{*}{ Brain region } & \multirow{2}{*}{$\mathrm{R} / \mathrm{L}$} & \multicolumn{3}{|c|}{ MNI } & \multirow{2}{*}{ Voxel } & \multirow{2}{*}{ Z-score } \\
\hline & & $x$ & Y & Z & & \\
\hline $\mathrm{CPL}$ & L & -30 & -69 & -24 & 89 & -5.1574 \\
\hline VMPFC & L & -3 & 54 & 33 & 169 & 8.021 \\
\hline OFC & L & -51 & 42 & -6 & 140 & 5.9012 \\
\hline RO & L & -60 & 6 & 6 & 232 & 9.109 \\
\hline PUT & $\mathrm{R}$ & 21 & -6 & -6 & 157 & 6.4611 \\
\hline CAU & L & -21 & -9 & 15 & 119 & -5.4231 \\
\hline PCC & L & -24 & -63 & 3 & 193 & -6.0579 \\
\hline HP & L & -27 & -12 & -9 & 51 & 5.2567 \\
\hline IC & L & -24 & 33 & 12 & 82 & -5.2257 \\
\hline IC & $\mathrm{R}$ & 36 & 3 & 21 & 69 & 4.3767 \\
\hline SMA & L & -21 & 0 & 33 & 473 & 8.5119 \\
\hline
\end{tabular}

FDR, false discovery rate; MNI, Montreal Neurological Institute. ACC, anterior cingulate cortex; CAU, caudate; CPL, cerebellum posterior lobe; FG, fusiform gyrus; HP, hippocampus gyrus; IC, insular; OFC, orbitofrontal cortex; PCC, posterior cingulate cortex; PHP, parahippocampal gyrus; PUT, putamen; RO, rolandic operculum; SMA, supplementary motor area; THS, thalamus; VMPFC, ventromedial prefrontal cortex. 

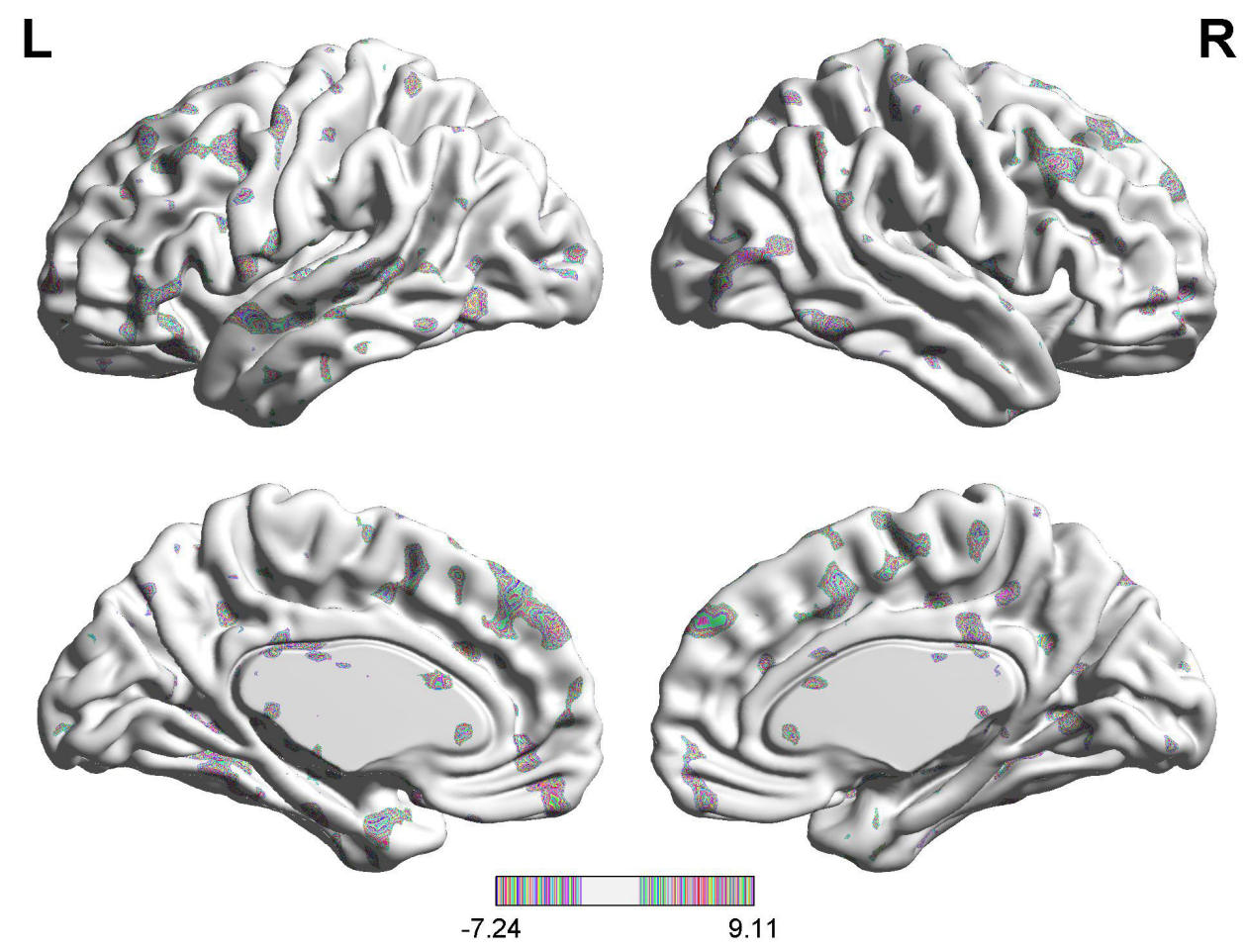

Figure S2 Brain areas showing significantly FC with the ACC in the placebo status in extrovert group. 


\section{Brain response of nocebo effect in introvert subjects (ROI-voxel)}

In the FC network of the ACC results, compared with the pain status, the nocebo status displayed increased $\mathrm{FC}$ in the angular gyrus (AG), amygdala (AMYG), CAU, and the
VMPFC. Nevertheless, the ACC exhibited significantly decreased $\mathrm{FC}$ in the $\mathrm{CPL}, \mathrm{FG}$, middle temporal lobe (MTL), superior temporal lobe (STL), pregenual anterior cingulate cortex (pgACC), DLPFC, and the primary somatosensory area (S1) (Table S3 \& Figure S3).

Table S3 The locations of the brain areas showing significantly $\mathrm{FC}$ with the ACC in the nocebo response in introvert group. $(\mathrm{P}<0.05, \mathrm{FDR}<0.05)$

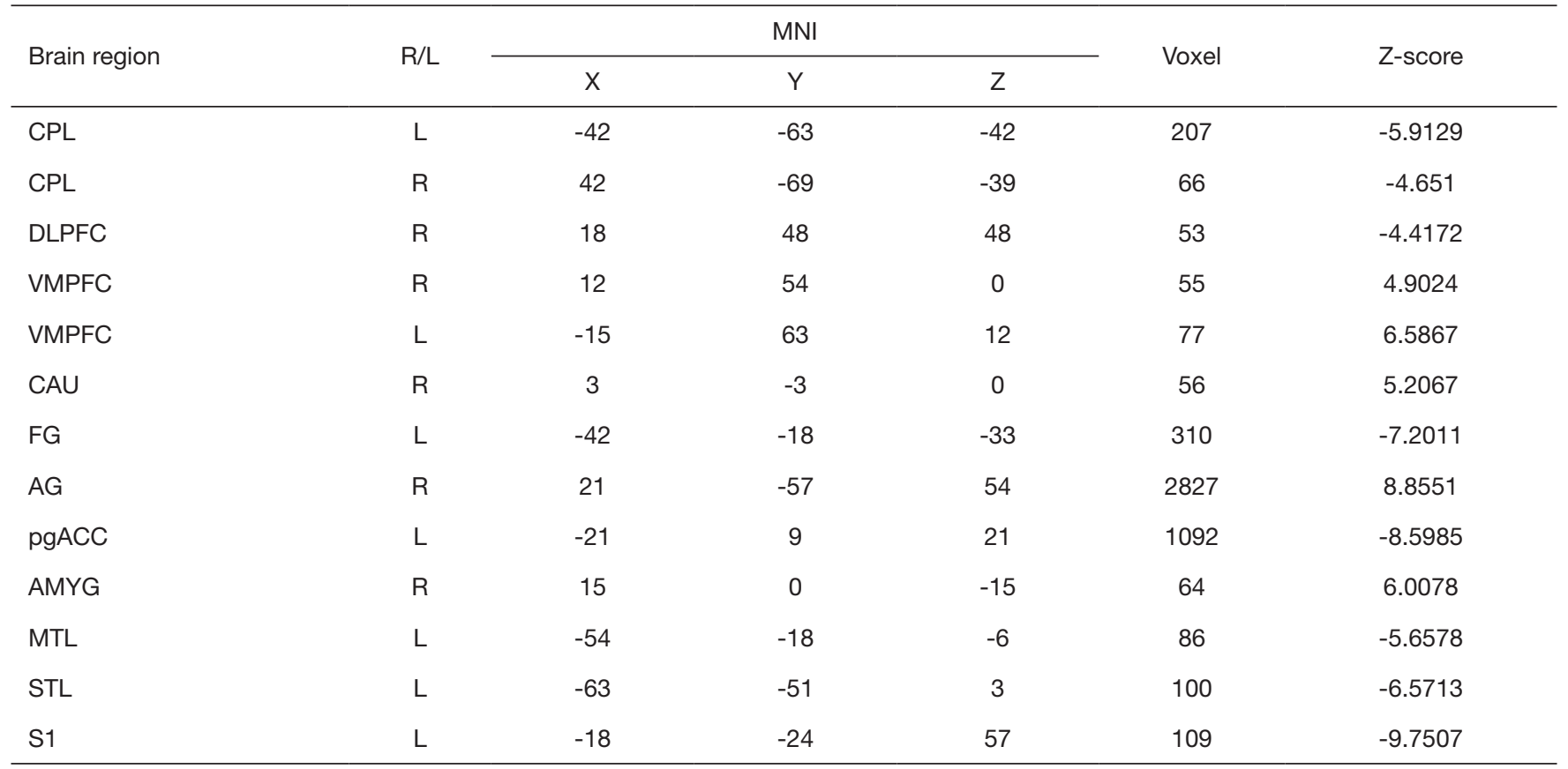

FDR, false discovery rate; MNI, Montreal Neurological Institute. ACC, anterior cingulate cortex; AG, angular gyrus; AMYG, amygdala; CAU, caudate; CPL, cerebellum posterior lobe; DLPFC, dorsolateral prefrontal cortex; FG, fusiform gyrus; MTL, middle temporal lobe; pgACC, pregenual anterior cingulate cortex; STL, superior temporal lobe; S1, primary somatosensory area; VMPFC, ventromedial prefrontal cortex. 

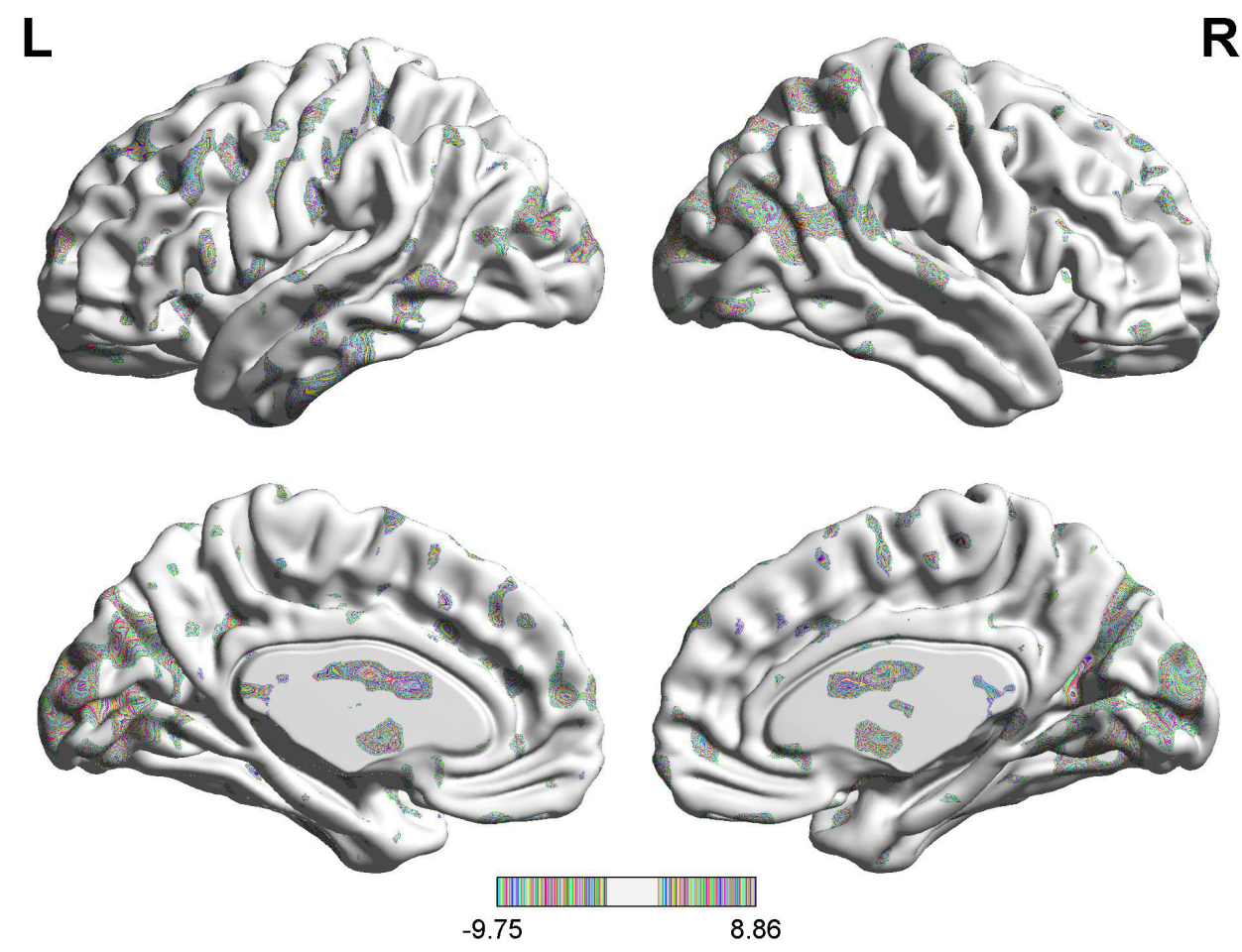

Figure S3 Brain areas showing significantly FC with the ACC in the nocebo status in introvert group. 


\section{Brain response of nocebo effect in extrovert subjects (ROI-voxel)}

In the FC network of the ACC results, compared with the pain status, the nocebo status displayed increased FC in the AMYG, OFC, AG, MTL, DLPFC, pgACC, prefrontal cortex (PFC) and S1. However, the ACC exhibited significantly decreased FC in the CPL, PHP, MTL, PCC, and the mid-cingulate cortex (MCC) (Table S4 \& Figure S4).

Table S4 The locations of the brain areas showing significantly FC with the ACC in the nocebo response in extrovert group. (P<0.05, FDR $<0.05$ )

\begin{tabular}{lcccccc}
\hline \multirow{2}{*}{ Brain region } & $\mathrm{R} / \mathrm{L}$ & \multicolumn{2}{c}{ MNI } & Voxel & Z-score \\
\cline { 3 - 5 } CPL & $\mathrm{L}$ & -24 & -66 & -24 & 186 & -6.757 \\
DLPFC & $\mathrm{R}$ & 42 & 27 & 36 & 246 & 7.1519 \\
PFC & $\mathrm{L}$ & -6 & 36 & 57 & 1031 & 7.5948 \\
OFC & $\mathrm{R}$ & 51 & 48 & -9 & 68 & 5.1296 \\
pgACC & $\mathrm{R}$ & 3 & 12 & 18 & 86 & 6.0886 \\
PCC & $\mathrm{L}$ & -15 & -33 & 30 & 240 & -6.1891 \\
MCC & $\mathrm{L}$ & -3 & -39 & 48 & 186 & -6.5768 \\
PHP & $\mathrm{L}$ & -15 & -81 & 36 & 1237 & -7.7407 \\
PHP & $\mathrm{R}$ & 21 & -48 & -9 & 53 & -4.7556 \\
AMYG & $\mathrm{R}$ & 30 & -3 & -12 & 96 & 5.695 \\
MTL & $\mathrm{L}$ & -42 & -66 & -3 & 79 & -5.3356 \\
MTL & $\mathrm{R}$ & 27 & 36 & 6 & 63 & 4.9623 \\
AG & $\mathrm{R}$ & 48 & -57 & 48 & 284 & 5.4723 \\
S1 & $\mathrm{L}$ & -15 & -33 & 69 & 106 & 5.7912
\end{tabular}

FDR, false discovery rate; MNI, Montreal Neurological Institute. ACC, anterior cingulate cortex; AG, angular gyrus; AMYG, amygdala; CPL, cerebellum posterior lobe; DLPFC, dorsolateral prefrontal cortex; MCC, mid-cingulate cortex; MTL, middle temporal lobe; OFC, orbitofrontal cortex; PFC, prefrontal cortex; pgACC, pregenual anterior cingulate cortex; PCC, posterior cingulate cortex; PHP, parahippocampal gyrus; $\mathbf{S 1}$, primary somatosensory area. 

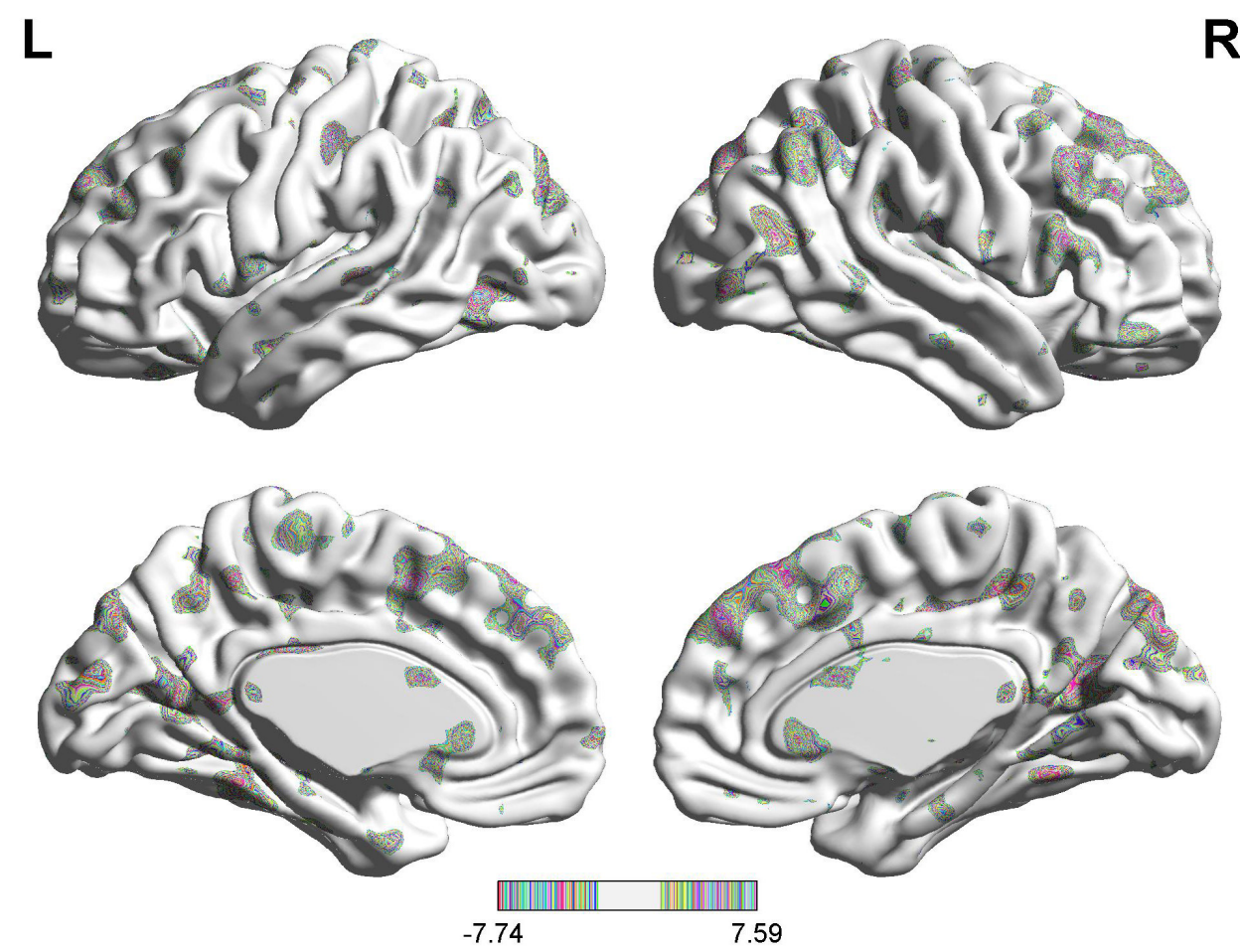

Figure S4 Brain areas showing significantly FC with the ACC in the nocebo status in the extrovert group. 